\section{The Impact of Transitional}

Movements and Non-Manual

Markings on the Disambiguation

of Locally Ambiguous Argument

Structures in Austrian Sign

Language (ÖGS)
Language and Speech

2019, Vol. 62(4) 652-680

(c) The Author(s) 2018

Article reuse guidelines: sagepub.com/journals-permissions DOI: 10.1 I77/00238309/880I399 journals.sagepub.com/home/las

@SAGE

\title{
Julia Krebs
}

University of Salzburg, Austria

\section{Ronnie B. Wilbur}

Purdue University, USA

\section{Phillip M. Alday}

Max Planck Institute for Psycholinguistics, The Netherlands

\section{Dietmar Roehm}

University of Salzburg, Austria

\begin{abstract}
Previous studies of Austrian Sign Language (ÖGS) word-order variations have demonstrated the human processing system's tendency to interpret a sentence-initial (case-) ambiguous argument as the subject of the clause ("subject preference"). The electroencephalogram study motivating the current report revealed earlier reanalysis effects for object-subject compared to subjectobject sentences, in particular, before the start of the movement of the agreement marking sign. The effects were bound to time points prior to when both arguments were referenced in space and/or the transitional hand movement prior to producing the disambiguating sign. Due to the temporal proximity of these time points, it was not clear which visual cues led to disambiguation; that is, whether non-manual markings (body/shoulder/head shift towards the subject position) or the transitional hand movement resolved ambiguity. The present gating study further supports that disambiguation in ÖGS is triggered by cues occurring before the movement of the disambiguating sign. Further, the present study also confirms the presence of the subject
\end{abstract}


preference in ÖGS, showing again that signers and speakers draw on similar strategies during language processing independent of language modality. Although the ultimate role of the visual cues leading to disambiguation (i.e., non-manual markings and transitional movements) requires further investigation, the present study shows that they contribute crucial information about argument structure during online processing. This finding provides strong support for granting these cues some degree of linguistic status (at least in ÖGS).

\section{Keywords}

Austrian Sign Language, gating study, subject preference, transitional movement, non-manuals, ambiguity resolution

\section{Introduction}

A previous event-related brain potential (ERP) experiment (Krebs, 2013, 2017; see also Krebs, Malaia, Wilbur, \& Roehm, 2018) investigating the processing of word order variations (subject/ object/verb (SOV) versus object/subject/verb (OSV) orders) in Austrian Sign Language (ÖGS) had revealed earlier disambiguation effects for OS compared to SO sentences at time points occurring before what is expected based on standard descriptions of the start of lexical signs. In particular, the ERP effects were observed before the start of the movement of the disambiguating agreement marking element. Because it is possible in sign languages to provide manual and non-manual information in parallel, it was difficult to determine which visual cue(s) might have indicated the argument structure to the viewer, that is, led to disambiguation. In the present study we further investigated when transitive ÖGS structures are disambiguated and which cues lead to ambiguity resolution on the behavioral level.

\section{I.I Simultaneity and sequentiality in sign languages}

When investigating the question of how and to what degree language modality may influence language structure and processing, the examination of sign languages (which are expressed in the visual-manual modality; that is, they use the three-dimensional signing space to convey linguistic information) plays an important role. Language modality influences the linguistic structure of sign and spoken languages in certain ways. The "extra" spatial dimensions of sign languages allow for a degree of simultaneity not possible with spoken language. Indeed, a number of descriptions of sign language grammars emphasize that sign language structures are organized more simultaneously compared to spoken languages, which are structured more serially (e.g., Bross \& Hole, 2017; Wilbur, 2000). In particular, in sign languages different pieces of information can be provided in parallel using the non-manual and manual channels for sign production, which both perform important grammatical functions and are generally independent from each other, despite temporal alignments at the prosodic level (for the sign level, see Wilbur, 2015; for the sentence level, Wilbur, 2018). In addition, the various articulators within each channel can provide different information simultaneously. Interestingly, this simultaneous realization of linguistic information, that is, the layering of non-manual with manual components, has been described for all grammatical levels in sign languages (e.g., Bross \& Hole, 2017; Wilbur, 2000).

Different non-manual articulators may perform different grammatical functions: for example, in American Sign Language (ASL), adverbial/adjectival marking is mostly expressed by the lower part of the face whereas clausal marking is mostly expressed by the upper face, allowing non-manual 
markings to be combined and layered. Thus it is possible to layer the non-manual yes-no question marking ("q," the underline shows its domain with respect to the manual signs) with an adverbial non-manual marking ("mm," "with enjoyment, easily") as in (1) (from Liddell, 1980, p. 44)1:

(1)

$$
\begin{gathered}
\text { MAN FISH [I: CONTINUOUs] } \\
\text { Is the man fishing with relaxation and enjoyment? }
\end{gathered}
$$

Furthermore, components of manual articulators can also be layered. For instance, the handshape of the verb GIVE, co-occurring with the expression via movement of the core act of giving, can mark a handling classifier which indicates that the given object is of a particular shape or grasped with a particular hand configuration (e.g., a round or a thin object). More complex information, including, for example, spatial arrangement/orientation in addition to the physical properties of an object, can also be expressed this way. This complex simultaneity with each manual articulator (hand) is further enhanced by the availability of two hands, which can independently indicate distinct objects and can in their interaction indicate the spatial relationship or interaction between those objects (although they are not completely free to act independently; Malaia, Borneman \& Wilbur, 2017). The pairing of manual articulators further enables the simultaneous expression of morphological information such as marking of number (Lepic, Börstell, Belsitzman \& Sandler, 2016). For instance, in sentences with one-handed agreeing verbs, duality of recipients can be marked in parallel by using two hands when, for example, each hand produces the agreeing verb but is directed towards different locations in space, instead of producing the verb with one hand twice in succession, once for each recipient (Klima \& Bellugi, 1979).

These differences in structural preferences for signed compared to spoken languages, that is, the tendency for simultaneously organized structures in sign languages and the preference for sequentially organized constructions in spoken languages, may be related to different properties of the visual and auditory modalities and their respective perceptual processing systems. In particular, the human visual processing system shows better discrimination of simultaneously presented information, whereas the auditory system shows better discrimination of sequentially ordered information. Nonetheless, the visual system also discriminates and processes sequentially presented information (and the auditory system can discriminate simultaneously presented information in a limited fashion).

Although studies on sign language grammar mostly emphasize the simultaneous nature of sign languages (particularly for forming lexical roots), there is also sequentiality involved (both derivationally and inflectionally; Wilbur, 2015). For instance, there are serially organized (affixal) structures in sign languages such as agentive marking in German Sign Language (adding the sign PERSON to a noun such as POLITICS for expressing the occupational title "politician"; Pfau \& Steinbach, 2013) or sequential reduplication affixing in ASL (Wilbur, 2009). ${ }^{2}$

Crucially, despite the fact that in sign languages more information can be presented (and processed) in parallel, sequential processing remains important as signs - like spoken words - unfold in time. As such, incremental processing remains important for sign language, but is more complex than incrementality in spoken language due to the more extensive multiplexing possible in the visual domain. Sequentiality within sign language processing has been demonstrated experimentally. For instance, with regard to the phonological parameters of signs, location ("place of articulation") is the parameter that is identified first (e.g., Emmorey \& Corina, 1990). In addition, a modality-specific aspect that may influence incremental sign language processing is the continuous visibility of the (non-)manual articulators during sign language perception to the addressee. Thus, the transitional trajectories between signs may provide important information for linguistic 
processing (e.g., Hosemann, Herrmann, Steinbach, Bornkessel-Schlesewsky, \& Schlesewsky, 2013; Jantunen, 2010; Ten Holt, van Doorn, de Ridder, Reinders \& Hendriks, 2009; Wilbur, 1990).

\section{I.2 Processing of locally ambiguous argument structures in spoken/written languages}

Research on language processing provides strong evidence that language is processed incrementally, that is, in a serial manner in that the processing system does not wait until the whole sentence is spelled out, but immediately takes into account the incoming input and integrates it with previously established context information (see e.g., Crocker, 1994; Frazier \& Fodor, 1978). During incremental processing, local ambiguity may arise, especially in verb-final languages with flexible word order and potentially ambiguous case marking. The sentences in $(2 \mathrm{a} / \mathrm{b})$ exemplify locally ambiguous argument structures in German. Both preverbal argument noun phrases (NPs), the proper name (Melanie) as well as the bare plural (Tänzerinnen), are case-ambiguous with regard to their syntactic function and the argument structure, that is, the syntactic relation of the arguments is ambiguous until the sentence-final verb disambiguates the sentence towards $\mathrm{SO}(2 \mathrm{a})$ or OS (2b):

(2a) [SO order $] \quad$ Ich glaube, dass Melanie Tänzerinnen kennt.

[I believe that MelanieNOM/ACC/DAT.SG dancersNOM/ACC/DAT.PL knowsSG] I believe that Melanie knows the dancers.

(2b) [OS order $] \quad$ Ich glaube, dass Melanie Tänzerinnen kennen.

[I believe that MelanieNOM/ACC/DAT.SG dancersNOM/ACC/DAT.PL knowPL] I believe that the dancers know Melanie.

For various spoken languages it has been shown that a sentence-initial (case-) ambiguous argument NP is preferentially interpreted as the subject of a sentence. This preference towards an SO interpretation leads to reanalysis in cases of locally ambiguous object-initial sentences. This reanalysis causes enhanced processing costs that are reflected in longer reading times (e.g., Schlesewsky, Fanselow, Kliegl \& Krems, 2000), lower acceptability ratings and longer reaction times (e.g., Bornkessel, McElree, Schlesewsky \& Friederici, 2004; Haupt, Schlesewsky, Roehm, Friederici \& Bornkessel-Schlesewsky, 2008), longer fixations as well as more regressions within reading (e.g., Kretzschmar, Bornkessel-Schlesewsky, Staub, Roehm \& Schlesewsky, 2012) and different ERP effects for OS compared to SO orders (see Bornkessel-Schlesewsky \& Schlesewsky, 2009 for an overview).

The preference for subject-initial structures, both with ambiguous case marking and more generally, is a very robust effect for which many explanations have been offered. However, explanations based on syntactic subjecthood, such as dependencies (Gibson, 1998) or structural position (Crocker, 1994), seem insufficient in light of similar phenomena in typologically diverse languages, such as Chinese, Japanese, Turkish and Hindi, where traditional definitions of "subject" break down (Keenan, 1976; Li \& Thompson, 1976). Therefore, within their so-called Extended Argument Dependency Model, Bornkessel and Schlesewsky (2006) proposed that the "subject preference" does not arise from any particular properties related to subjecthood per se but rather is an epiphenomenon of a general least-effort processing strategy in that the processing system attempts to generate less complex or minimal structures ("the MINIMALITY principle"; Bornkessel \& Schlesewsky 2006). Further, Bornkessel-Schlesewsky, Choudhary, Witzlack-Makarevich, and Bickel (2008) suggested that the "subject preference" is a universal ambiguity resolution processing strategy that is applied whenever an ambiguous argument NP is encountered. 


\section{I.3 Argument relations in sign languages}

In sign languages, transitive argument structures can be expressed by word order, animacy or verb agreement, which is a modification to the verb form to reflect its arguments (Klima \& Bellugi, 1979; Liddell, 1980; Sandler \& Lillo-Martin, 2006). If word order is used in a language to indicate argument structure, the subject and object argument(s) appear in a specific sentence position, which is usually the basic word order of the particular language. In ASL, for instance, the basic sign order is SVO.

In languages in which animacy can be used to mark argument structure, the animacy of the involved arguments in relation to the subcategorization properties of the verb are crucial for determining who is doing what to whom. For example, in ASL a transitive sentence with an animate argument, an inanimate argument, and a verb that takes an animate argument such as DRINK is unambiguously understood even when the sentence has a non-basic SOV order (Fischer, 1975). For describing the process of sign language verb agreement, the mechanisms of spatial referencing of discourse participants need to be introduced. Within sign language discourse, referents may be associated with specific locations in space by non-manual (e.g., body shift, eye gaze) and/or manual means (e.g., index/pointing signs), especially if the signer expects to refer back to them again. Referencing may be used for physically present as well as non-present referents. The signer can re-reference a previously introduced discourse participant by pointing back to previously established locations (among other mechanisms). The established spatial relation between a referent and a location will hold until the location is re-associated with another discourse referent or when a new topic is introduced. Full agreeing verbs realize agreement by starting their movement from the location associated with the subject argument and ending at the location of the object (Fischer, 1975; Padden, 1983). In addition, hand orientation may also be modulated, with the palm and/or fingertips directed towards the object position, that is, facing towards the object (Brentari, 1989). Such hand orientation (i.e., "facing") may co-occur with movement, but may also be the only indicator of agreement, depending on the formation of the verb. There is also a set of irregular agreeing verbs that show a reversed movement from the object to the subject position, but which may also show facing towards the object (Meir, 1998). ${ }^{3}$

Furthermore, there are also verbs that do not use movement to show agreement, the "plain" verbs. Interestingly, sign languages differ in how they indicate argument relations in sentences with plain verbs. Some sign languages use a strict word order to avoid ambiguity in such structures (e.g., ASL or British Sign Language; Fischer, 1975), whereas others may make use of independent agreement marker (AgrM) signs. AgrMs are manual signs that show movement (like regular agreeing verbs) from subject to object position and may also show facing (for more detailed information on AgrMs in different sign languages see e.g., Sapountzaki, 2012; Steinbach \& Pfau, 2007).

Finally, there are also sign-language-specific means to unambiguously indicate argument structure. For example, Geraci (2013) proposed a "default referencing" for Italian Sign Language in that subjects are always referenced at the ipsilateral side of the signer and objects are placed contralaterally (if the spatial relationship between the arguments themselves does not need to be expressed). It is not known how widespread such a strategy might be among existing sign languages.

Although there have been some claims that case marking as defined for some spoken languages may exist in sign languages as well (e.g., Kegl, 2004, assumes that ASL uses a system of case marking for pronominalization), there is now some consensus among linguists that sign languages lack grammatical case marking (Sandler \& Lillo-Martin, 2006).

Thus, descriptions of sign language verb systems propose that in sentences with an agreeing verb or an AgrM, the movement and/or the facing of the agreement marking element indicates the relation between the arguments. This implicitly assumes that the preverbal part of a transitive 


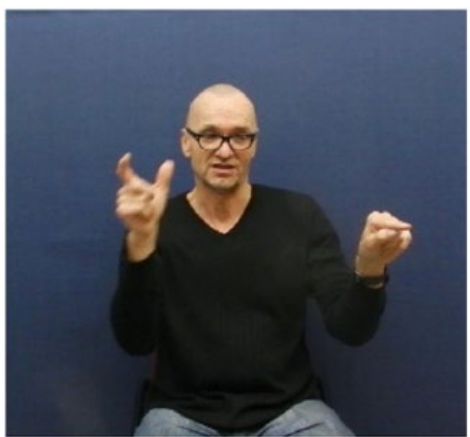

(A)

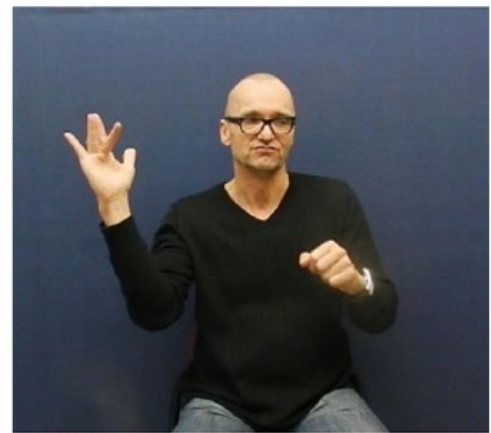

(B)

Figure I. Two ÖGS agreement markers (A) AgrM-BC is produced with the Baby-C handshape; (B) AgrM-MF is generated by a forward-pointing middle finger.

AgrM: agreement marker.

structure is locally ambiguous in verb-final sign languages (like ÖGS) that allow different orderings of the preverbal arguments.

ÖGS has SOV as its basic sign order (Skant et al., 2002; Wilbur, 2002, 2005), but can have nontopicalized OS orders in contexts with agreeing verbs and AgrMs accompanying plain verbs. For ÖGS, two forms of AgrMs have been described. AgrM-BC is produced with a Baby-C handshape and AgrM-MF is formed with a forward-pointing middle finger (Figure 1). Both signs move from the location of the subject to that of the object and show (palm) facing towards the object. Both ÖGS AgrMs can appear before and after the verb as well as in sentence second position after the subject (for more information about the AgrMs used in ÖGS, see Krebs, Wilbur \& Roehm, 2017).

Like for other sign languages, there are, so far, no descriptions of ÖGS which report any overt markers that indicate an argument's syntactic function. In addition, there does not appear to be any default referencing in ÖGS such as that which has been reported for Italian Sign Language (Geraci, 2013).

\section{I.4 Processing of argument relations in ÖGS_-previous ERP results}

To investigate the processing of locally ambiguous argument structures in ÖGS, we previously conducted an ERP study using sentences with SO and OS orders involving either agreeing verbs or plain verbs accompanied by a preverbal AgrM (Table 1).

The participants, all Deaf ÖGS signers $(n=16)$, watched ÖGS real-time videos and judged the acceptability of each sentence (for a more detailed description of the ERP study on ÖGS that built the basis of the present gating study, see Krebs, 2013, 2017). ${ }^{4}$ This study showed statistically significant lower acceptability ratings, longer reaction times, and an ERP effect for the OS orders in contrast to the SO orders. This pattern of results was observed for both verb types. ${ }^{5}$

Crucially, the ERP effects occurred in relatively early time windows, namely before the time point which is assumed-based on our current understanding of sentence structure in sign languages - to indicate the argument structure in sentences with agreeing verbs/AgrMs (i.e., the path movement and/or the facing of the agreement marking element). In particular, the effects were bound to the time point when both arguments were referenced in space and/or at the start of the 
Table I. Example sentences of the four experimental conditions.

\begin{tabular}{|c|c|c|}
\hline & SO & OS \\
\hline $\begin{array}{l}\text { Plain } \\
\text { verbs }\end{array}$ & $\begin{array}{l}\text { GIRL IX }{ }_{3 A} \text { WOMAN IX }{ }_{3 B}{ }_{3 A} A_{G R} M_{3 B} \\
\text { KNOW } \\
\text { The girl knows the woman. }\end{array}$ & $\begin{array}{l}\text { GIRL IX }{ }_{3 A} \text { WOMAN IX }{ }_{3 B}{ }_{3 B} A_{G R} M_{3 A} \\
\text { KNOW } \\
\text { The woman knows the girl. }\end{array}$ \\
\hline $\begin{array}{l}\text { Agreeing } \\
\text { verbs }\end{array}$ & $\begin{array}{l}\text { GRANDCHILD IX }{ }_{3 \mathrm{~A}} \\
\text { GRANDMOTHER IX } \mathrm{X}_{3 \mathrm{~B}}{ }_{3 \mathrm{AVISIT}} \mathrm{B} \\
\text { The grandchild visits the grandmother. }\end{array}$ & $\begin{array}{l}\text { GRANDCHILD IX }{ }_{3 A} \\
\text { GRANDMOTHER IX }{ }_{3 B}{ }_{3 B} V_{I S I T}{ }_{3 A} \\
\text { The grandmother visits the grandchild. }\end{array}$ \\
\hline
\end{tabular}

Note: Plain and agreeing verbs were presented in SO as well as OS orders. Signs are glossed with capital letters. Subscripts indicate reference points within signing space.

SO: subject/object; OS: object/subject; IX: manual index sign; AgrM: agreement marker.

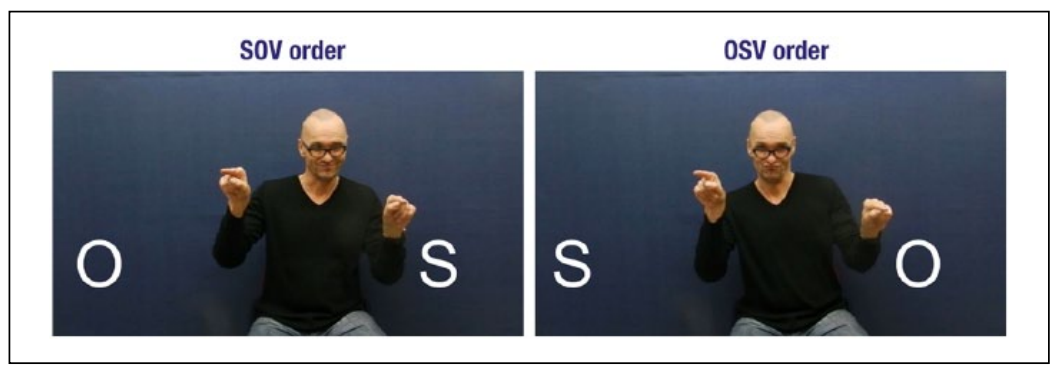

Figure 2. Illustration of the body shift towards the position associated with the subject. This body shift was observed in $\mathrm{SO}$ as well as OS sentences.

SOV: subject/object/verb; OSV: object/subject/verb; SO: subject/object; OS: object/subject.

transitional movement towards the phonological specifications of the disambiguating sign. ${ }^{6}$ Due to the fact that the duration between these two time points is very short, it was not possible to determine whether the effects were bound to the time point when the arguments were referenced in space or when the hand starts the transitional movement towards the agreement marking element. ${ }^{7}$

Both of these time points constitute possible cues for triggering disambiguation. On the one hand, the transitional movement towards the agreement marking element may already indicate from where the disambiguating sign's path movement will start. On the other hand, visual inspection of the stimulus material revealed that in the majority of the sentences the signer uses nonmanual markings, namely body/shoulder or head shift towards the subject position, during referencing of the second argument NP, that is, before any transitional movement towards the disambiguating sign (see Figure 2 for an illustration of the body shift towards the position associated with the subject). ${ }^{8}$ This body/shoulder/head shift was present in SO as well as OS orders. Thus, these non-manual markings may also have induced disambiguation in that the body/shoulder/head shift towards the subject position indicates which element is the subject (Krebs, 2017; see also Krebs et al., 2018). ${ }^{9}$

Thus, both the layering of manual and non-manual components as well as the visibility of transitional movements between signs may enable signers to extract information about the argument structure before the agreement marking element starts its movement.

However, given how short the duration was from the time point when both arguments were referenced in space to the transitional movement of the hand towards the agreement marking element, 
it was not clear which of these cues led to disambiguation. This issue was further investigated in the present study.

\section{I.5 The present study}

In order to better determine the time point of disambiguation we used the gating method, in which $\mathrm{SO}$ and OS orders were presented by successively prolonging gates to Deaf ÖGS signers. To date, there have been only a few studies investigating sign language using the gating method. The previous studies have mainly focused on lexical access of isolated signs (Emmorey \& Corina, 1990; Grosjean, 1981; Morford \& Carlson, 2011) or of lexical access of signs presented within a sentence context (Clark \& Grosjean, 1982). In the present study, however, the gating method was used to investigate the time course of syntactic processing of ÖGS word order variations. The main goal of the study was to test when signers can identify the syntactic function of the arguments during incremental language processing and which visual cues trigger this identification process. In addition, this study should provide converging evidence for the claim of a subject preference in ÖGS as observed in the previous ERP study (see also Krebs et al., 2018).

Under the assumption of locally ambiguous argument structures and a "subject preference" in ÖGS, we hypothesized that, first, the signers cannot identify the syntactic function of the arguments until both argument NPs have been introduced and indexed; and second, prior to the time point of disambiguation (during the presentation of both argument NPs), the majority of the sentences will be judged to be SO. Although it was an open question which visual cues would trigger reanalysis on the behavioral level, we assumed - in line with the previous ERP study - an earlier time point of disambiguation than what would be expected from the general definition of sign "onset" in sign language linguistics. In particular, we hypothesized that the structures may be disambiguated before the movement of the agreement marking element started.

\section{Method}

\section{I Materials}

For the gating study, a set of sentences from the stimulus material filmed for the earlier ERP study (Krebs, 2013, 2017) was presented in successive gates. The first gate was defined as the sequence from video onset to the onset of the second argument NP. For the purposes of constructing the gate, "sign onset" was defined as the time point when the phonological target parameters handshape and location were established. Each subsequent gate was prolonged by four frames (frames per $\mathrm{s}=$ 29.97; time between two gates $=133.5 \mathrm{~ms}$ ) until the movement of the disambiguating agreeing verb ended or until the onset (time point when its target handshape reached its target location) of the plain verb after the disambiguating AgrM in the plain verb condition. Thus, this last gate represented the whole stimulus sentence. The sentences consisted of between 10 and 19 gates (see Figure 3).

For this procedure, 20 plain verb and 20 agreeing verb constructions were presented in two order conditions (SO/OS). Subjects saw every verb in both word order conditions. The overall verb types (agreeing verb vs. plain verb) can be divided into further subcategories. The plain verb condition comprised sentences with two different AgrMs (AgrM-BC and AgrM-MF). The agreeing verb condition involved two different forms of referencing of the argument NPs: serial referencing (the index sign referencing the first argument was not held in space during signing of the second argument NP) and simultaneous referencing (the index sign referencing the first argument was held in space during signing of the second argument NP). In both plain verb subcategories, the referencing 


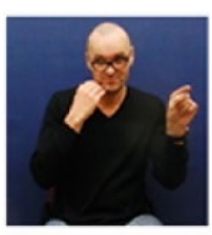

Gate 01

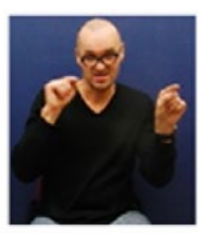

Gate 02

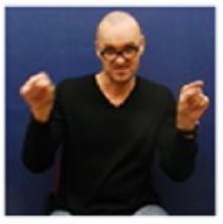

Gate 03

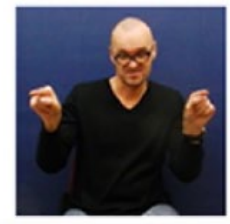

Gate 04

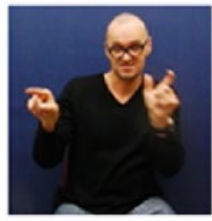

Gate 05

Figure 3. Gating procedure.

Gate I constitutes the interval from video onset to the onset of the second argument. Each subsequent gate was prolonged by four frames.

was the same; that is, in both, simultaneous referencing was present. In the following, we analyze both the overarching distinction between plain and agreeing verbs as well as the more refined distinctions among all four subcategories.

\subsection{Procedure}

Prior to the start of the trials, instructions were shown as an ÖGS video signed by one of the authors. To get the attention of the participants, every trial started with the presentation of a fixation cross on the screen for $2000 \mathrm{~ms}$, followed by an empty black screen for $200 \mathrm{~ms}$. Then the first gate was presented. The videos were presented in the middle of the screen with an image size of 1280 $\times 720$. After each gate, a green question mark appeared for $3000 \mathrm{~ms}$. On seeing this question mark, the subjects had to indicate by button press which of the two arguments was the active one, that is, most likely to be the subject. Thus, they had to decide whether they thought that either the argument introduced first or the argument introduced second was the active one. If they were not sure, they had to guess. Button location (left, right) for active argument was counterbalanced across subjects. If the subjects did not respond before the question mark disappeared, the next gate started automatically. The gated sentences were presented in a single block from one of four pseudo-randomizations. Before starting the actual experiment, a training session (involving four example sentences; two SO and two OS orders, respectively) was presented to give the subjects experience with the task requirements and to ask questions in case anything was unclear. The whole experiment lasted about 60-90 min. The participants were paid $20 €$ per session.

\subsection{Participants}

In total, 14 Deaf participants (nine women) from 23 to 66 years $($ mean $=38.5, S D=12.2$ ) took part in the study. The majority were born deaf or lost their hearing very early in life. Most of them acquired ÖGS starting at the age from 4 to 7. All participants had hearing parents and most of them came from the area of Salzburg.

\subsection{Data analysis and results}

Initial summary statistics revealed that the vast majority of sentences, both SO and OS sentences, were initially (i.e., after the first gate) judged to be SO. Indeed half of the participants rated the first gate of every single sentence (whether SO or OS) consistently as SO order, whereas the other half of the subjects rated a small set of sentences as OS structure after seeing the first gate. Out of a total 


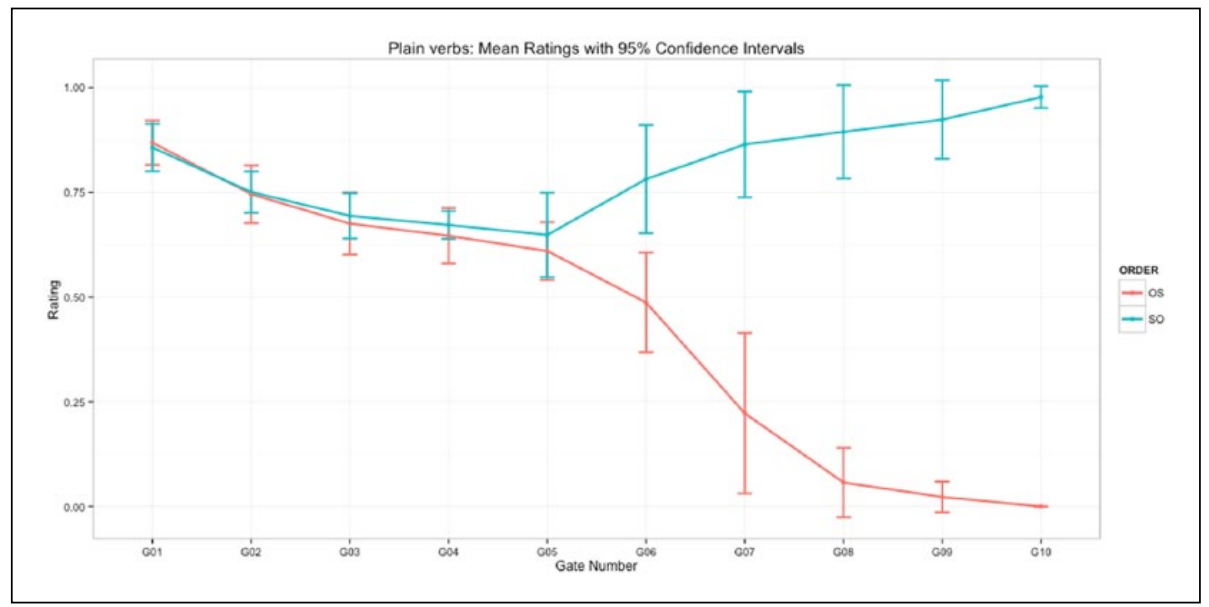

Figure 4. Comparison of $\mathrm{SO}$ and $\mathrm{OS}$ orders for the plain verbs.

Gate numbers are given on the $x$-axis; mean ratings, that is, the proportion of sentences interpreted as $\mathrm{SO}$, are presented on the $y$-axis. The decisive gate appeared at gate 6 .

SO: subject/object; OS: object/subject.

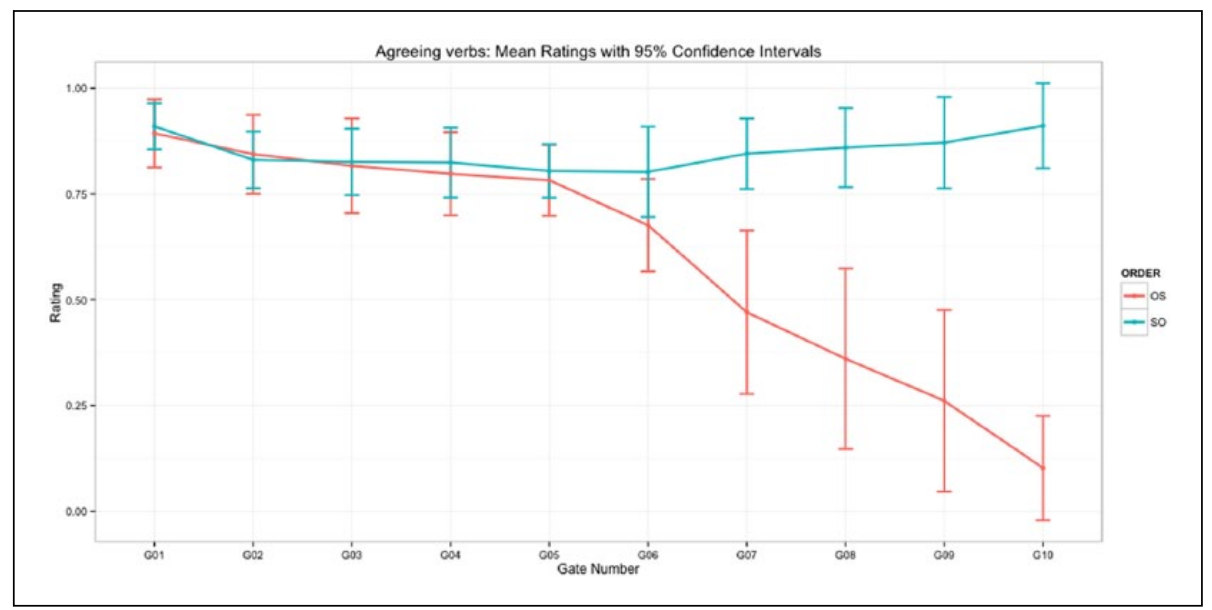

Figure 5. Comparison of SO and OS orders for the agreeing verbs.

Gate numbers are given on the $x$-axis; mean ratings, that is, the proportion of sentences interpreted as $\mathrm{SO}$, are presented on the $y$-axis. The decisive gate appeared at gate 7 .

SO: subject/object; OS: object/subject.

of 280 OS sentences presented over all subjects and all conditions, only 30 were rated initially as OS structures (11\%), whereas $250(89 \%)$ were initially rated as SO.

For a more detailed analysis of the time course of interpretation, we used logistic mixed-effects regression (see also Jaeger, 2008) over all gates; that is, over the entire time course. An alternative analysis using statistics computed at each gate is presented in Figures 4 and 5 to give the reader an overall impression of the time course and its variability. However, this gate-wise analysis is not discussed in more detail in the text as the difference between "significant" and "not significant" is 
not, itself, statistically significant (Gelman \& Stern, 2006) and it is thus difficult to make inferences about the time course without a combinatorial explosion in the number of pairwise comparisons necessary.

The random effects structure consisted of by-item and by-participant terms. The by-item terms included the intercept and slopes for gate and word order. This models variation in the baseline interpretation by lexical item and any effects of particular word-order realization as well as the speed at which the interpretation converges to the final interpretation. Allowing for a by-item variable speed also compensates for differences in sentence duration (in terms of absolute time), with the fixed effect for gate then representing the "average" timing effect across stimuli. The by-participant terms included the intercept and a slope for word order, allowing for variation in the baseline strength of the hypothesized subject preference and resulting issues in processing alternative word orders. This parsimonious model structure supports the experimental hypotheses in line with recent proposals for random-effects structure (see also Bates, Kliegl, Vasishth \& Baayen, 2015) and avoids overparameterizing the model on a small dataset (e.g., as would be the case with the "maximal" structure proposed by Barr, Levy, Scheepers \& Tily, 2013). Models were fit with the Laplace Approximation for Maximum Likelihood using the lme4 package (Bates, Mächler, Bolker \& Walker, 2015).

The individual sentences varied somewhat in length and thus had a slightly different number of gates (see above). The mixed-effects analysis was restricted to the first 13 gates, as half of the stimulus set had at least that many gates. The remaining variation in length is captured in the model by the by-item slope for gate.

Fixed-effect predictors beyond gate and word order were either verb type (plain vs. agreeing) or subcategory (AgrM-BC and AgrM-MF for plain verbs, simultaneous and sequential referencing for agreeing verbs). All categorical predictors were encoded with sum coding; gate was treated as a continuous predictor (which is not unreasonable, given that each gate except the first and last represented an identical length of time). Subject-initial interpretations were encoded as 1 and object-initial as 0 in the dependent variable; thus positive regression coefficients can be interpreted as the (log) odds ratio in favor of the subject-initial interpretation, whereas negative coefficients reflect $(\log )$ odds ratio in favor of the object-initial interpretation.

2.4.I Statistical analysis by verb type. For the analysis by verb type (see Table 2 and Figure 6 ), we find significant main effects for gate, verb type and word order as well as interactions for gate with verb type, gate with word order and a three-way interaction between gate, verb type and word order.

Additionally, the intercept is significant and strongly positive, which indicates a strong preference for subject-initial interpretations in the absence of further information. The main effect for gate simply indicates that the interpretation changed with increasing information and is not of further interest (in particular, the subject-initial interpretation becomes less likely with additional, possibly contrary information). The main effect for verb type indicates that agreeing verbs were more likely than the plain verbs to receive a subject-initial interpretation across all time points. The main effect for word order indicates that OS sentences were more likely than SO sentences to be interpreted as SO. This seems counterintuitive at first; however, there is an interaction between gate and word order in the opposite direction, and main effects may be misleading in the presence of interactions. In particular, the interaction goes in the opposite direction, with each additional gate halving the odds that an OS sentence will be interpreted as SO. The final interactions between gate and verb type and among gate, verb type and word order are much weaker, but indicate that the interpretation of agreeing verbs was less strongly influenced by each additional gate than that of plain verbs. 
Table 2. Summary of model fit by verb type.

$\begin{array}{lllll}\text { AIC } & \text { BIC } & \frac{\text { logLik }}{4953} & \frac{\text { Deviance }}{3919} & \frac{\text { df.resid }}{6658}\end{array}$

Scaled residuals:

\begin{tabular}{cccccc} 
Minimum & IQ & Median & $3 Q$ & Maximum \\
\hline-16.95 & -0.15 & 0.09 & 0.31 & 139.21
\end{tabular}

Random effects:

\begin{tabular}{lllrrr} 
Groups & Term & SD & \multicolumn{2}{c}{ Correlation } \\
\hline Item & (Intercept) & I.137868 & \\
& & word.order[OS] & 0.69732 I & 0.749 & \\
VP & Gate & (Intercept) & 0.095967 & -0.888 & -0.827 \\
& Number of obs: 6675, groups: Itemnr, I7; VP, I4.
\end{tabular}

Fixed effects:

\begin{tabular}{|c|c|c|c|c|c|}
\hline & Estimate & SE & $z$ value & $\operatorname{Pr}(>|z|)$ & \\
\hline (Intercept) & 3 & 0.55 & 5.5 & $3.3 e-08$ & $* * *$ \\
\hline Gate & -0.3 & 0.03 & -10 & $1.8 \mathrm{e}-24$ & $* * *$ \\
\hline verb.type[agreeing] & 1.1 & 0.15 & 7.4 & $1.5 \mathrm{e}-13$ & $* * *$ \\
\hline word.order[OS] & 1.7 & 0.24 & 7.1 & $1.5 e-12$ & 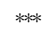 \\
\hline Gate:verb.type[agreeing] & -0.065 & 0.02 & -3.3 & 0.001 & $* *$ \\
\hline Gate:word.order[OS] & -0.54 & 0.018 & -29 & $2.3 e-189$ & $* * *$ \\
\hline $\begin{array}{l}\text { verb.type[agreeing]:word. } \\
\text { order[OS] }\end{array}$ & 0.1 & 0.12 & 0.85 & 0.4 & \\
\hline $\begin{array}{l}\text { Gate:verb.type[agreeing]:word. } \\
\text { order[OS] }\end{array}$ & 0.033 & 0.015 & 2.2 & 0.026 & $*$ \\
\hline
\end{tabular}

Note: Generalized linear mixed model fit by maximum likelihood (Laplace Approximation). Family: binomial, link: logit. AIC: Akaike Information Criterion; BIC: Bayesian Information Criterion; logLik: log Likelihood; df.resid: residual degrees of freedom; SD: standard deviation; OS: object/subject; VP: participant; obs: observations; Itemnr: Item number; SE: standard error.

$*_{p}<0.05$, **p $<0.01$, ***p $<0.001$.

From both our modeled effects and our aggregate ratings plots, it is clear that early on a subjectinitial interpretation was preferred. The strength of this preference decreased with increasing information until about gate 6 in the plain verb condition and gate 7 in the agreeing verb condition, at which point the preference for SO or OS interpretation started converging towards the actual word order, as seen in Figures 4 and 5. This is reflected in the effect plot (Figure 6) in the broad confidence intervals until about gate 7 for the SO word order

2.4.2 Statistical analysis by subcategory. Further dividing the verb types into subcategories, we find a main effect for gate, subcategory, and word order as well as two-way interactions for gate with subcategory and gate with word order and three-way interactions for gate and subcategory with word order (see Table 3 and Figure 7). The effect for the intercept and gate are interpreted as above. For the subcategories, we find that AgrM-BC and AgrM-MF - the plain verb subcategories - are less likely than average (more precisely: than the grand mean over all conditions) to be interpreted 


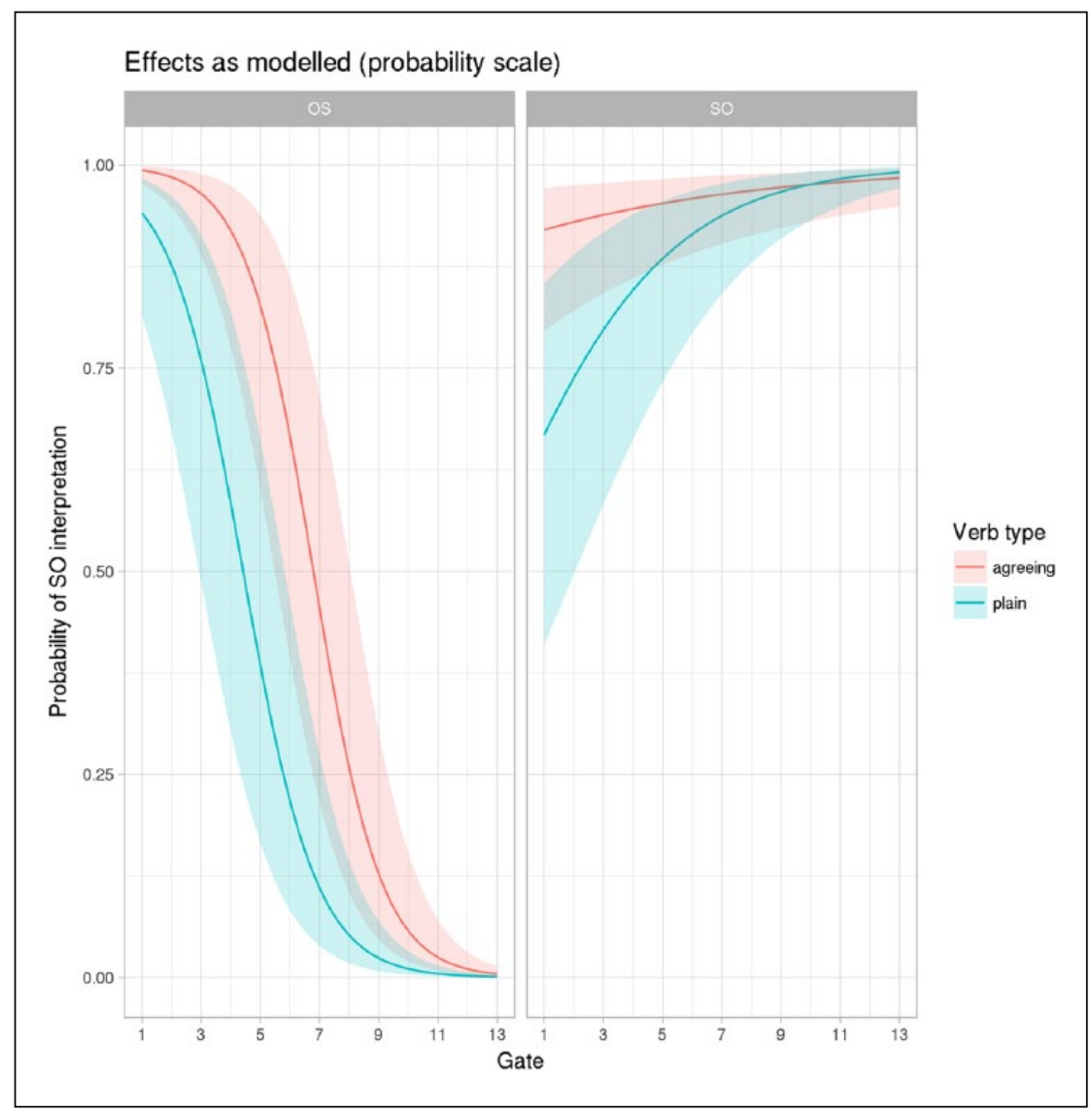

Figure 6. Effects as modeled for OS and SO orders by verb type.

These represent the time courses that the model extracted from the data. Shaded areas reflect $95 \%$ confidence intervals. The low probability of SO interpretation in plain verbs with SO word order is a result of response variability and reflected in the width of the confidence interval (which overlaps with the confidence interval from the gate-wise grand averages presented in Figure 4).

OS: object/subject; SO: subject/object.

as SO, whereas agreeing verbs with serial referencing are more likely than average. ${ }^{10}$ This is in line with the effects observed above for the plain versus agreeing verb contrast, albeit with more subtlety. The main effect for word order as well as its interaction with gate have nearly identical estimates and nearly identical interpretations to the model above. Similarly, the interaction between subcategory (serial referencing category) and gate follows the interaction above between gate and verb type.

More interesting in this model are the three-way interactions. These interactions show that the different subcategories converge to the (correct) OS interpretation in OS sentences at different rates; in other words, the subcategories are differentially sensitive to additional information.

Finally, a comparison of log likelihood, AIC, and BIC suggests that the model relying on the more fine-grained subcategories rather than the coarser verb types provides a better description of the data. This is particularly revealing for the serial referencing subcategory, which requires the longest to diverge from the initial SO interpretation (only reaching 50\% OS interpretation at gate 9 for OS sentences). 
Table 3. Summary of model fit by subcategory.

$\frac{\text { AIC }}{3922} \frac{\text { BIC }}{4092} \frac{\text { logLik }}{-1936} \frac{\text { Deviance }}{3872} \frac{\text { df.resid }}{6650}$

Scaled residuals:

\begin{tabular}{cccccc} 
Minimum & IQ & Median & $3 Q$ & Maximum \\
\hline-13.28 & -0.14 & 0.09 & 0.3 & 110.44
\end{tabular}

Random effects:

\begin{tabular}{llllll} 
Groups & Term & SD & \multicolumn{2}{c}{ Correlation } \\
\hline Itemnr & (Intercept) & 0.834479 & \\
& & Gate & 0.073168 & -0.762 & \\
& & word.order[OS] & 0.482224 & 0.496 & -0.645 \\
VP & $\quad$ (Intercept) & 1.73755 I & & \\
& word. order[OS] & 0.460254 & -0.029 \\
Number of obs: 6675, groups: Itemnr, I7; VP, I4.
\end{tabular}

Fixed effects:

\begin{tabular}{|c|c|c|c|c|c|}
\hline & Estimate & SE & $z$ value & $\operatorname{Pr}(>|z|)$ & \\
\hline (Intercept) & 3 & 0.52 & 5.8 & $5.2 \mathrm{e}-09$ & $* * *$ \\
\hline Gate & -0.3 & 0.026 & -12 & $8.9 e-31$ & *** \\
\hline subcategory[AgrM-BC (plain)] & -0.78 & 0.29 & -2.7 & 0.0068 & $* *$ \\
\hline subcategory[AgrM-MF (plain)] & -1.1 & 0.32 & -3.5 & 0.00039 & $* *$ \\
\hline subcategory[serial (agreeing)] & 1.7 & 0.3 & 5.6 & $1.9 \mathrm{e}-08$ & $* *$ \\
\hline word.order[OS] & 1.8 & 0.21 & 8.4 & $4.6 \mathrm{e}-17$ & $* * *$ \\
\hline Gate:subcategory[AgrM-BC (plain)] & 0.046 & 0.036 & 1.3 & 0.2 & \\
\hline Gate:subcategory[AgrM-MF (plain)] & 0.052 & 0.038 & 1.4 & 0.18 & \\
\hline Gate:subcategory[serial (agreeing)] & -0.11 & 0.035 & -3.1 & 0.0022 & $* *$ \\
\hline Gate:word.order[OS] & -0.56 & 0.019 & -29 & $7.9 e-189$ & $* *$ \\
\hline $\begin{array}{l}\text { subcategory[AgrM-BC } \\
\text { (plain)]:word.order[OS] }\end{array}$ & -0.15 & 0.2 & -0.72 & 0.47 & \\
\hline $\begin{array}{l}\text { subcategory[AgrM-MF } \\
\text { (plain)]:word.order[OS] }\end{array}$ & 0.058 & 0.23 & 0.25 & 0.8 & \\
\hline $\begin{array}{l}\text { subcategory[serial } \\
\text { (agreeing)]:word.order[OS] }\end{array}$ & 0.13 & 0.22 & 0.6 & 0.55 & \\
\hline $\begin{array}{l}\text { Gate:subcategory[AgrM-BC } \\
\text { (plain)]:word.order[OS] }\end{array}$ & 0.044 & 0.025 & 1.8 & 0.076 & . \\
\hline $\begin{array}{l}\text { Gate:subcategory[AgrM-MF } \\
\text { (plain)]:word.order[OS] }\end{array}$ & -0.1 & 0.028 & -3.7 & 0.00018 & $* * *$ \\
\hline $\begin{array}{l}\text { Gate:subcategory[serial } \\
\text { (agreeing)]:word.order[OS] }\end{array}$ & 0.12 & 0.024 & 4.9 & $9.3 e-07$ & $* * *$ \\
\hline
\end{tabular}

Note: Generalized linear mixed model fit by maximum likelihood (Laplace Approximation). Family: binomial, link: logit. AIC: Akaike Information Criterion; BIC: Bayesian Information Criterion; logLik: log Likelihood; df.resid: residual degrees of freedom; SD: standard deviation; Itemnr: Item number; OS: object/subject; VP: participant; obs: observations; SE: standard error; BC: Baby-C handshape; MF: forward-pointing middle finger.

*p-value $<0.05 ;{ }^{* *} p$-value $<0.01 ;{ }^{* * * *} p$-value $<0.00 \mathrm{I}$.

2.4.3 Individual item analysis. To further specify which information within the visual signal may have led to the divergence in ratings between both orders (SO vs. OS), that is towards OS, an individual item analysis was carried out. In particular, the critical gate was taken to be the gate at 


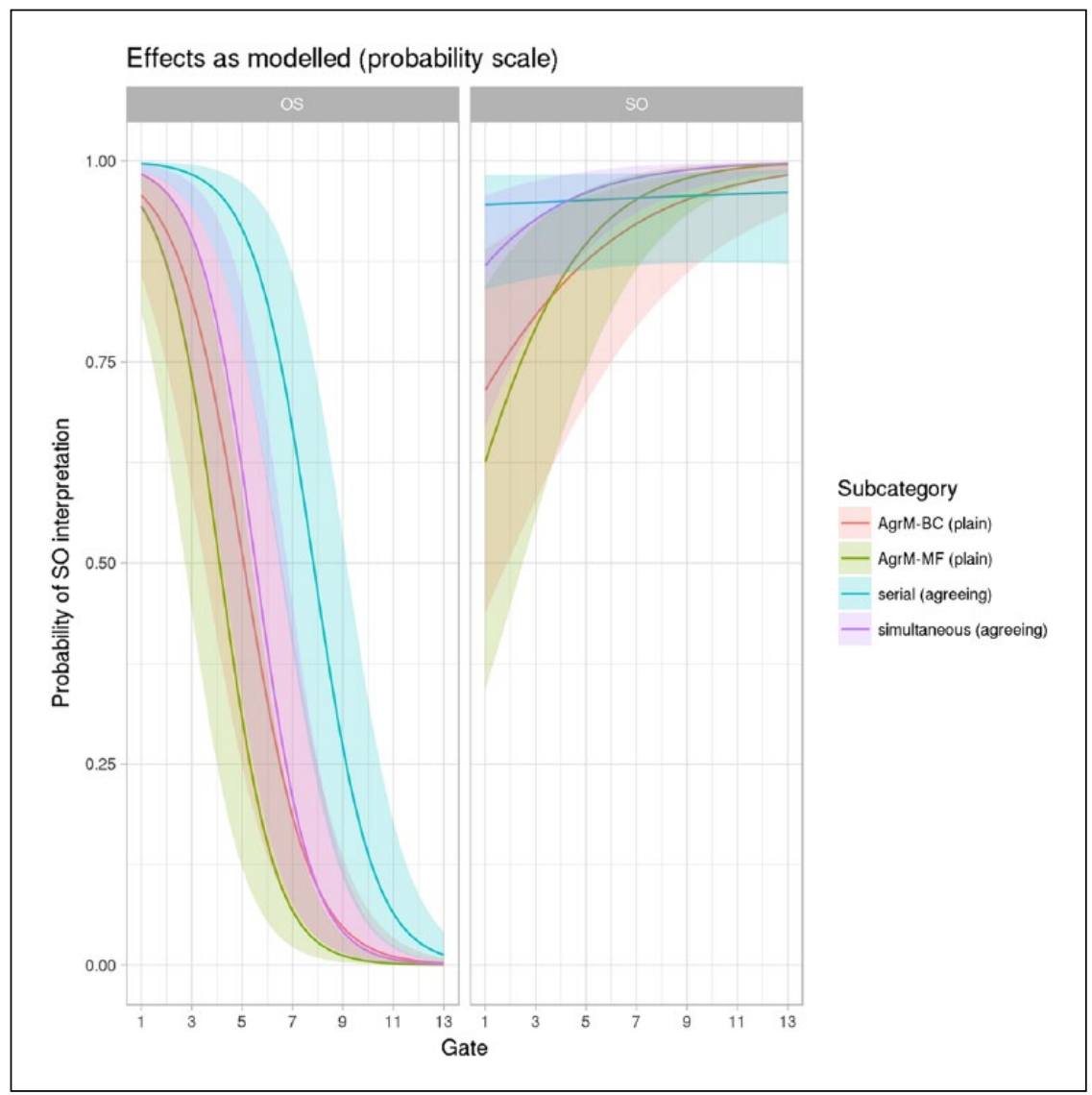

Figure 7. Effects as modeled for OS and SO orders by subcategory.

As in Figure 6, these represent the time courses that the model extracted from the data. Shaded areas reflect $95 \%$ confidence intervals. The plain verb subcategories are initially less likely to be interpreted as SO than agreeing verbs with serial referencing are. Different subcategories converge to the (correct) OS interpretation in OS sentences at different rates. The serial referencing subcategory requires the longest to diverge from the initial $\mathrm{SO}$ interpretation.

OS: object/subject; SO: subject/object; AgrM: agreement marker; BC: Baby-C handshape; MF: forward-pointing middle finger.

which the OS order falls below a 50\% chance of being interpreted as SO order. This is the gate at/ immediately after the point where the orangish-red line crosses the dotted red line in Figure 8.

Several cues unfolding successively in time were considered as potentially deciding factors. These relevant cues consisted of the following time points:

(a) before the index referencing the second argument reaches its target location;

(b) when the index referencing the second argument reaches its target location;

(c) when the hand that produces the movement of the disambiguating agreement marking element slightly starts its trajectory movement towards its target location and the hand still shows the index handshape (early transition phase, TP);

(d) when the handshape changes towards the target handshape of the disambiguating sign, but the target handshape has not been fully established (medial TP);

(e) when the target handshape of the disambiguating sign is established, but has not reached the target location/orientation present at the initiation of the movement of the disambiguating sign (late TP); 


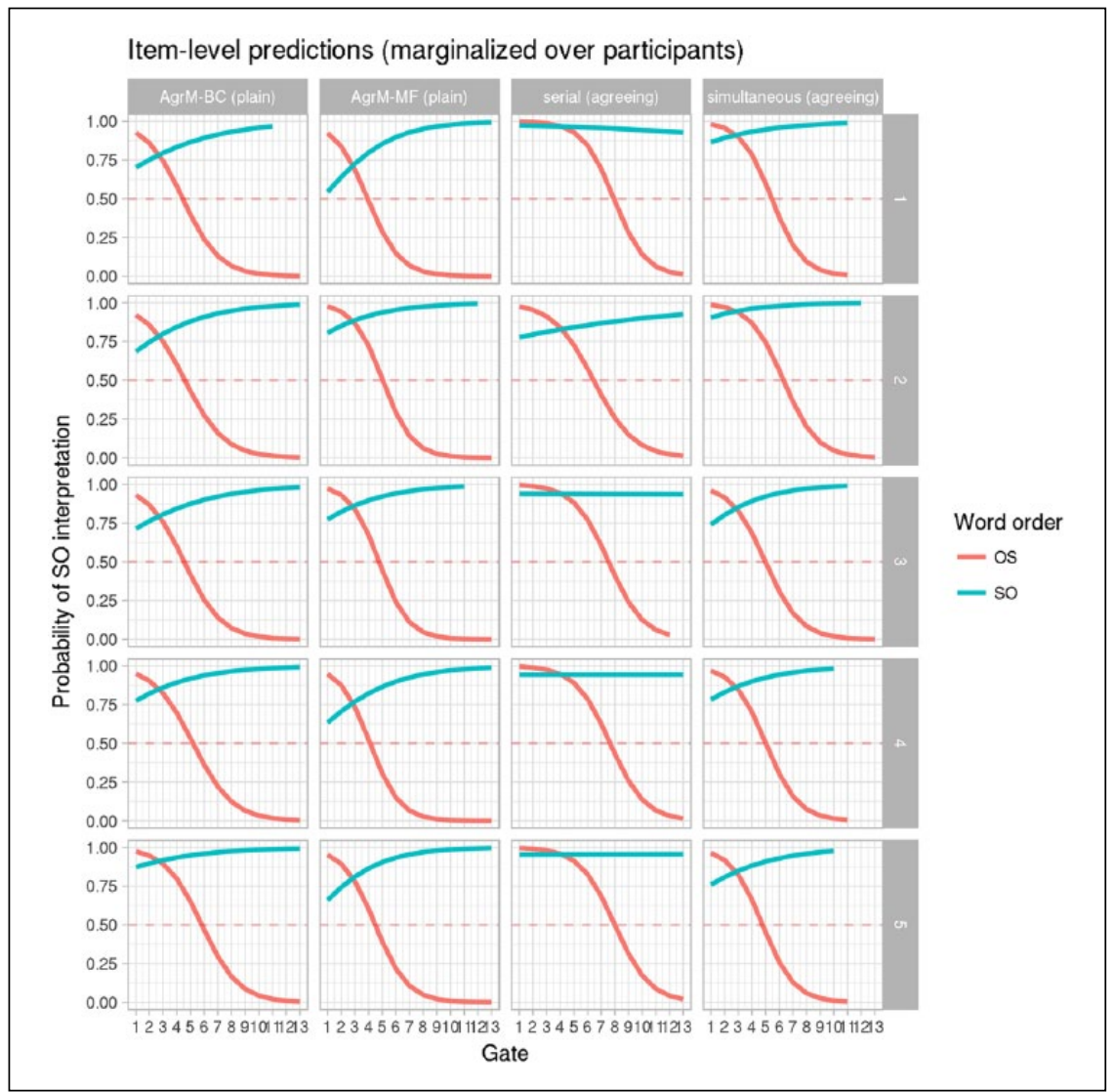

Figure 8. Comparison of $\mathrm{SO}$ and $\mathrm{OS}$ orders for the individual items per subcategory.

Gate numbers are given on the $x$-axis; ratings as modeled are presented on the $y$-axis. As in Figure 6, these are the time courses as extracted by the model, that is, predicted by the fitted statistical model. Marginalizing over participants yields time courses for an abstract, "average" participant. The critical gate was taken to be the gate at which the OS order falls below a $50 \%$ chance of being interpreted as SO order, represented here by the dotted line.

SO: subject/object; OS: object/subject.

(f) when the target handshape of the disambiguating sign has reached its target location ("first hold" in terms of the "Hold-Movement-Hold" model of Liddell \& Johnson, 1989) from where the movement of the disambiguating sign starts; and

(g) when the movement of the disambiguating sign starts (see Figures 9 and 10). Table 4 gives an overview of the relevant gates and the visual cue presented in this critical gate for the individual items per verb type and per subcategory.

The individual item analysis revealed that in all items the first critical gate, that is, the gate at which the OS orders have less than $50 \%$ chance of being interpreted as SO orders, can be observed before the movement of the disambiguating sign started. In the majority of sentences the critical gate was observed during the referencing of the second argument $(n=10)$ or when the second argument was referenced by an index sign $(n=2)$. Further, in a number of items the critical gate was revealed within the early transitional phase $(n=7)$. In only one item (belonging to the agreeing verbs: serial referencing subcategory) was the target location of the agreeing verb reached at the critical gate. 


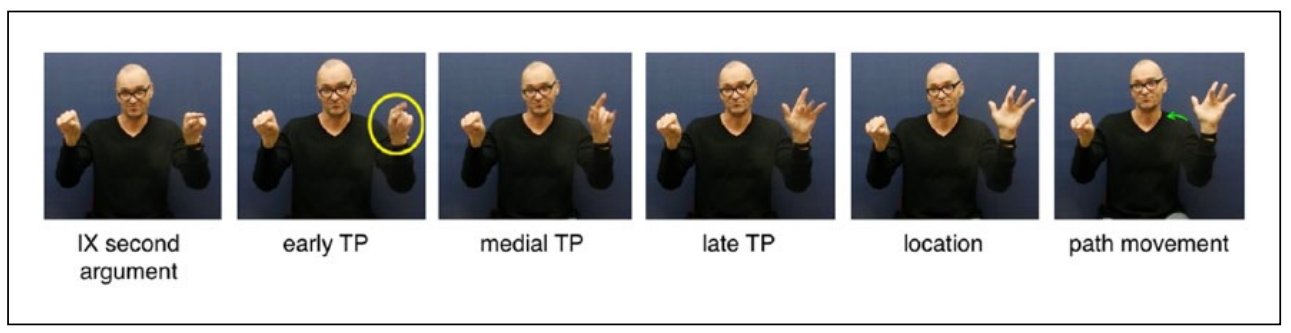

Figure 9. The specific time points used for describing the cue(s) observed in the gates at which the first significant drift occurred.

IX: manual index sign; TP: transition phase.

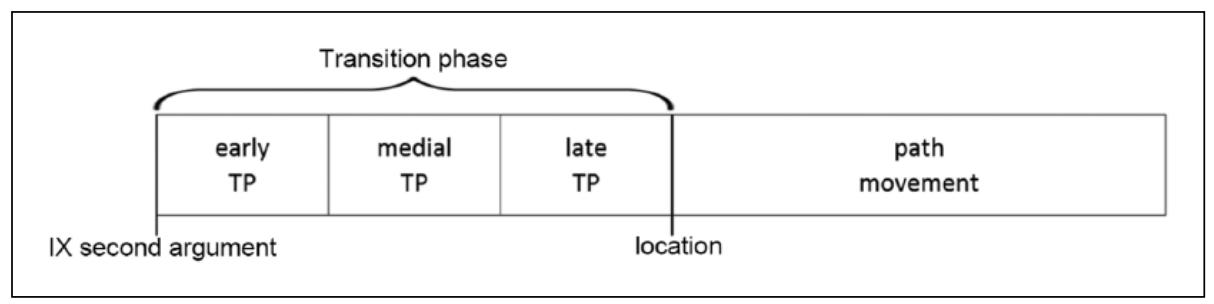

Figure 10. Schematic illustration of the various time points for describing the cue(s) observed in the gates at which the first significant drift occurred.

IX: manual index sign; TP: transition phase.

\section{Discussion}

\section{I Transitional movements and non-manual markings resolve syntactic ambiguity}

We observed visual cues preceding the start of the movement of the disambiguating sign in every item at the critical gate. This finding contrasts with the general assumption within the sign language literature that the movement and/or the facing of the agreement marking element is the cue that indicates the argument structure (e.g., Friedman, 1976; Pfau \& Steinbach, 2006). Hence, the present study shows that more information is presented earlier than previously thought, and signers actually use this information in sentence comprehension.

In a number of the OS sentences the early transitional movement of the hand towards the disambiguating sign was present at the critical gate. Thus, the slight movement of the index hand away from its final position often in combination with a body shift towards the subject position resolved ambiguity. In most of the sentences, however, the critical gate was observed during the referencing of the second argument or when both arguments were referenced in space. Visual inspection of the video material suggests that in these sentences, non-manual markings co-occurring with the index referencing the second argument may have provided crucial information about the argument structure. These non-manual markings were either a body/shoulder/head shift and/or a more pronounced face/chin/eye gaze position towards the subject location.

In particular, in cases in which the critical gate occurred when the second argument was referenced, a (start of a) body shift towards the subject position (the signers' right side in OS) was visible during the referencing of the second argument. This body shift towards the subject position was also present in many of the items in which the early transitional phase was visible at the critical 
Table 4. Individual item analysis per verb type and per subcategory.

Item Critical Visual cue observed at the critical gate for OS orders gate Preceding IX NP2 IX NP2 Early TP Medial TP Late TP Location Path movement

Plain verbs: AgrM-BC

\begin{tabular}{|l|l|l|l|l|l|l|l|l|}
\hline 1 & 5 & & & $x$ & & & & \\
\hline 2 & 5 & $x$ & & & & & & \\
\hline 3 & 5 & & & $x$ & & & & \\
\hline 4 & 6 & & & $x$ & & & & \\
\hline 5 & 6 & $x$ & & & & & & \\
\hline
\end{tabular}

Plain verbs: AgrM-MF

\begin{tabular}{|l|l|l|l|l|l|l|l|l|}
\hline 1 & 4 & $\mathrm{x}$ & & & & & & \\
\hline 2 & 5 & & $\mathrm{x}$ & & & & & \\
\hline 3 & 5 & & & $\mathrm{x}$ & & & & \\
\hline 4 & 5 & $\mathrm{x}$ & & & & & & \\
\hline 5 & 5 & $\mathrm{x}$ & & & & & & \\
\hline
\end{tabular}

Agreeing verbs: serial referencing

\begin{tabular}{|l|l|l|l|l|l|l|l|l|}
\hline 1 & 8 & & & $x$ & & & & \\
\hline 2 & 7 & $x$ & & & & & & \\
\hline 3 & 8 & & & & & & $x$ & \\
\hline 4 & 8 & & & $x$ & & & & \\
\hline 5 & 8 & $x$ & & & & & & \\
\hline
\end{tabular}

Agreeing verbs: simultaneous referencing

\begin{tabular}{|l|l|l|l|l|l|l|l|l|}
\hline 1 & 6 & & & $x$ & & & & \\
\hline 2 & 7 & $x$ & & & & & & \\
\hline 3 & 5 & $\mathrm{x}$ & & & & & & \\
\hline 4 & 5 & & $\mathrm{x}$ & & & & & \\
\hline 5 & 5 & $\mathrm{x}$ & & & & & & \\
\hline
\end{tabular}

Note: The critical gate (i.e., the gate at which the OS order has less than 50\% chance of being interpreted as SO order) and the visual cue observed at the particular gates are presented.

IX: manual index sign; NP2: noun phrase 2; TP: transition phase; AgrM: agreement marker; BC: Baby-C handshape; MF: forward-pointing middle finger; OS: object/subject; SO: subject/object.

gate. In the other items without a body movement towards the subject position at the critical gate, the face/chin and sometimes the eye gaze of the signer was directed to the subject position. In contrast, in the corresponding SO orders the signers' chin/face was mostly positioned more centrally and the eye gaze was sometimes directed to the addressee. Even when the signers' chin/face was directed toward the argument referenced second (as in OS), the chin/face towards the second argument was mostly produced in a more reduced form and/or the body was simultaneously directed to the subject position (to the left in SO).

The observation that both non-manual markings co-occurring with the index referencing the second argument and that the early transition phase towards the disambiguating sign may provide 
information about argument structure supports the claim of reanalysis ERP effects observed by Krebs (2013, 2017; see also Krebs et al., 2018). These effects were bound to the index referencing the second argument and/or the transitional movement towards the disambiguating sign.

Furthermore, there was one item for which the critical gate was not observed until the location of the disambiguating sign was clearly established (item 3 of the agreeing verbs: serial referencing condition). The reason for this "later" occurrence of the critical gate may be semantic bias given the arguments themselves. The sentence involved the argument ordering MOTHER and CHILD, with the sign CHILD produced with the manual sign for BABY and the mouthing /kind/ ("child"). Thus, we assume that the first argument "MOTHER" was strongly preferred as the subject of the clause, because this argument is more likely to be the active one with respect to possible transitive relations between these two arguments. This strong preference for a subject-initial interpretation caused by this semantic bias might have led to a later abandonment of the subject preference.

\subsection{Providing converging evidence for the "subject preference"}

The observation that the majority of sentences $(89 \%)$ were initially interpreted as SO structures confirms the assumption of a subject preference in ÖGS. Only a small subset of subjects had initial OS interpretations in some conditions, and these did not occur in a systematic fashion, suggesting random error (or single-item semantic bias, as discussed above). The significant intercept in the statistical models also supports the claim of a subject preference. Interestingly, the data suggest that simultaneous referencing (holding the index referencing the first argument in space) does not lead to strengthening of the subject preference effect, that is, that holding the index referencing the first argument in space might make the first argument more likely to be the most salient argument- the subject. If this were the case, we would have expected a drop in ratings in the agreeing verbs condition with sequential referencing at the early gates compared to the simultaneously referenced agreeing verbs. However, just the opposite occurs: the serial referencing condition yields higher ratings in the first gates for both orders (SO and OS) compared to the simultaneously referenced agreeing verb condition. As outlined earlier, we did observe such a drop in the plain verb condition for those sentences of both subcategories that show simultaneous referencing. Thus, if simultaneous referencing were to lead to a stronger subject preference, we would not expect to have seen such a drop in ratings; that is, a drop in the tendency towards SO interpretation, in the early gates in the plain verb condition (see the Appendix for the plots of the ratings per subcategory).

\subsection{No case marking on the argument NPs}

The present study supports the assumption that ÖGS does not have any overt case marking on the argument NPs and thus that the sentences under examination truly involve local ambiguity. The fact that the participants were unable to identify the syntactic role of the first argument (i.e., which could be the subject or the object of the sentence) after the first argument was presented indicates that there seems to be no cue on the first argument and/or its corresponding index sign that could function as a kind of disambiguating case marker. Thus, if there were any (non-)manual cue(s) functioning as a form of case marker in ÖGS, signers would have been able to rate the sentences correctly after seeing the first gate. However, the critical gate was not observed until the second argument was referenced or when the hand that produced the movement of the disambiguating sign started its transitional movement. This finding supports the general theoretical claim that there are (at least in the ÖGS material used in this study) no forms of case marking on the preverbal argument NPs. Nevertheless, whether there are other (non-)manual markings that may function as a 
kind of case marker possibly within other constructions or grammatical environments has to be tested in further studies.

\subsection{Similarities and differences observed for the subcategories}

Interestingly, the gating experiment revealed a very similar pattern of results across the four subcategories with respect to the visual cues observed at the critical gates. That is, despite the fact that in the agreeing verb condition with serial referencing the critical gate appeared at a later time point compared to the other subcategories (on average, two gates later than the agreeing verbs involving simultaneous referencing), nonetheless similar visual cues were observed in the critical gates in the four subcategories.

The observation that agreeing verbs with serial referencing required the longest to diverge from the initial SO interpretation (only reaching 50\% OS interpretation on average at gate 9 for OS sentences) can be explained by properties of the stimulus material. In this subcategory, in three out of five sentences compound argument NPs that take longer to produce were involved. In contrast, in the other subcategories either no compound signs (AgrM-MF category) or only one compound was involved (AgrM-BC and simultaneous referencing categories). The "compounds" involved in the AgrM-BC and simultaneous referencing categories, however, differ with respect to those involved in the agreeing verbs serial condition. The compounds used in the agreeing verbs serial condition were true compounds consisting of two independent signs (e.g., MIT+ARBEITER"employee," KRANKEN+SCHWESTER - “nurse," KAMERA + MANN—“cameraman”). The complex signs used in the two other subcategories (AgrM-BC and simultaneous referencing) were instead both cases of female gender marking added to the signs for DAUGHTER and SECRETARY. This gender-marking morpheme is treated as an affix and is usually produced very fast. Thus, gender-marked NPs are not full compounds and do not take as much time, which may have led to the later presentation of information indicating the argument structure in sentences involving compounds. Therefore, we assume that the compounds involved in most of the items with serial referencing led to the later occurrence of the critical gates within this subcategory. ${ }^{11}$ This assumption is supported by the fact that for the two items involving the gender-marking sign the critical gate was observed earlier than for the structures with compounds. In particular, for both of these items (AgrM-BC category: item 2; simultaneous referencing category: item 3) the critical gate occurred at gate 5 . At the critical gate, the index referencing the second argument was not fully established, and thus non-manual markings provided important information with respect to the argument structure. Thus, although the visual cues presented at the critical gate are similar in the sentences with compounds and with the gender-marking signs, the critical gate for the sentences with compounds appeared later.

Therefore, we assume that the compound signs involved in most of the items in the serial referencing condition led to the fact that the serial referencing condition requires the longest to diverge from the initial SO interpretation compared to the other subcategories. The observation that similar cues led to disambiguation in the different subcategories support this assumption, that is that the difference in sign length leads to later critical gates in the serial condition and not the form of serial referencing per se. If this were the case, we would expect to observe that cues occurring later in time lead to the critical gate in all of the sentences with serial referencing.

The observation of similar results for the four subcategories at least with respect to the visual cues observed at the critical gate suggests that these structures were processed linguistically in a similar fashion independent of verb type, independent of referencing (simultaneous vs. serial) and independent of the AgrM. Whether the form of referencing as well as the verb type may influence (the time course of) disambiguation of locally ambiguous argument structures in ÖGS within other 
constructions or signed by a different signer, as well as in other sign languages, would have to be separately tested.

\subsection{Linguistic status of disambiguation cues?}

As pointed out before, in all of the items in the present study, the critical gate was observed before the time point usually considered as the marker indicating the argument structure in sentences with agreeing verbs and AgrMs (i.e., before the movement). Further, in all itemsexcept one - the critical gate occurred even before the time point that has been traditionally defined as "sign onset." 12 In particular, non-manual markings and transitional movement disambiguated the structures. As outlined below, the status of these cues within sign language grammar is unclear; they could be argued to be functional morphemes (again, possibly case) or syntactic cues that precede the onset of the lexical verb or AgrM. However, despite the uncertainty of their exact status, the fact that they contribute crucial information about argument structure during online processing provides strong support for granting them some degree of linguistic status (at least in ÖGS).

3.5.I Non-manual markings. That non-manuals may (optionally) mark syntactic agreement in sentences with different verb types has been described for ASL. Head tilt towards the subject position and eye gaze towards the object position were suggested as the primary AgrMs (Neidle, Kegl, MacLaughlin, Bahan \& Lee, 2000). In addition, body/shoulder/head shift towards the subject position marking subject agreement and body lean towards the object position marking object agreement were observed (Bahan, 1996). Further, the non-manuals reach their target position before the onset of the verb in ASL (an SVO language) and co-occur with the verb and the object. In an eyetracking study, Thompson, Emmorey, and Kluender (2006) confirmed the claims of Neidle et al. (2000) at least with regard to object marking by eye gaze towards the object in sentences with agreeing verbs. Thompson et al. (2006) also interpreted object agreement marking by eye gaze as syntactic agreement marking.

Whether the non-manual cues observed in many of the ÖGS orders at the critical gate constitute independent syntactic cues that function as a form of functional morpheme (such as a case or AgrM) in ÖGS is an open question. The fact that the non-manuals do not show a clear on- and offset, which has been described as a characteristic of syntactic non-manual markings (e.g., Liddell, 1980), speaks against the assumption that the non-manuals function as a functional morpheme. Thus, whether these non-manuals should be interpreted at the syntactic level, or whether they may rather constitute an instantiation of indexing (referencing back to the subject argument NP), needs further investigation.

3.5.2 Transitional movement. So far, most of the phonological models of sign languages (e.g., Brentari, 1998; Liddell \& Johnson, 1989; Sandler, 1989) explicitly or implicitly defined "sign onset" as the time point when the sign shows its "first hold" in space, that is when the target handshape slightly stops at the target position from where the movement will start (if the sign shows a path movement or an internal movement) or at the target position at which the sign is articulated (if the sign does not show a specific movement). ${ }^{13}$ In line with this definition a sign involving a path movement, such as an agreeing verb or AgrM, starts when the target handshape reaches the location from where its path movement starts. ${ }^{14}$

Further, older models of sign language phonology analyzed signs in a sentence as separated by "transitional movements" between them. As a result of this view, the "sign" has been described as the phase from the "first hold" to the "final hold" of a sign and the phases between the final hold 
of one sign and the first hold of the next sign were considered as transitional or trajectory movements (Liddell \& Johnson, 1989).

Brentari (1998) distinguished phonetic low-level transitional movement from phonological lexical movement. Lexical movement is one of the major phonological parameters of signs. Transitional movements, on the other hand, are low-level phonetic movements and have been treated as trajectories without any lexical meaning. There are clear differences between lexical and transition movement in that the articulatory production of transitions involves less muscular activity/stiffness than the production of lexical signs (Wilbur, 1990), the speed of lexical signs is slower and varies to a lesser degree than that of transitions, and lexical signs involve more accelerating and varying motion (Jantunen, 2013).

Brentari, Poizner, and Kegl (1995) reported that handshape changes within transitions were executed more abruptly than within lexical movements. Whereas $89.1 \%$ of the lexical movement was needed to execute lexical handshape changes, only $35.7 \%$ of the transitional movement was needed for producing transitional handshape changes. Brentari et al. (1995) argued that this statistic functions as a diagnostic to distinguish movements that are "phonetic level requirements" and movements that are "the result of an underlying distinctive feature specification."

Although most phonological models for sign languages adopted the "first hold" for defining the beginning of a sign, the "holds" of signs seem not to be an appropriate phonological cue for defining lexical sign onset and are better treated as phonetic level phenomena (Wilbur \& Allen, 1991; Wilbur \& Petersen, 1997). For example, holds that occur in signs are modulated by signing rate (Wilbur, 1990, 2009) and discourse situation (Wilbur \& Nolen, 1986). In addition, not every sign shows a clearly defined first hold (Hanke, Matthes, Regen, \& Worseck, 2012). In fact, in an analysis of 886 signed syllables taken from three Deaf signers in conversation with each other, only $16(1.8 \%)$ had clear, measurable onset holds; in contrast, 384 (43.2\%) were lexical movement only, and 114(12.8\%) were transitional movement only; ${ }^{15}$ the rest were combinations with initial and/or final holds (Wilbur \& Nolen, 1986).

There is empirical evidence suggesting that transitions as defined in the traditional sense have been underestimated in that they are neither meaningless nor "non-significant" phonetic trajectories. For example, transitions may be modulated in that they can take over properties typical for lexical movements to indicate stress marking in ASL (Wilbur, 1990). Furthermore, transitions may provide information about the upcoming lexical sign, as has been shown by studies of the recognition of isolated signs (Ten Holt et al., 2009) as well as by investigations of lexical access of signs presented in sentence context (Jantunen, 2010). In addition, transitions have been claimed to present important information during predictive processing (Hosemann et al., 2013).

There are a number of researchers who have discussed the status of transitions within sign language not only from a theoretical perspective, and have provided empirical evidence for the relevance of "transitions" within sign language processing (Green, 1984; Hosemann et al., 2013; Jantunen, 2010, 2015; Ten Holt et al., 2009; Wilbur, 1990). However, very few of these studies critically questioned the traditional definitions of sign onset and transitional movements (but see Green, 1984; Jantunen, 2010, 2015; Ten Holt et al., 2009).

Due to the facts that (1) the border between lexical and transitional movement is not as clearcut as has been proposed by sign language phonological models (Green, 1984; Jantunen, 2013; Ten Holt et al., 2009), (2) transitions may acquire properties typical for lexical movement (e.g., Wilbur, 1990) and (3) the trajectory movements towards and/or between signs may also provide important information for linguistic processing (Hosemann et al., 2013; Jantunen, 2010, 2015; Ten Holt et al., 2009; Wilbur, 1990), it seems plausible that transitions or parts of them may be part of lexical signs. Therefore, it is possible that they should not, strictly speaking, be considered as transitional, but possibly (earlier parts of) the lexical sign itself that provide relevant cue(s) for linguistic processing. This is a definitional problem that has so far not been faced for practical applications. 


\subsection{Age of acquisition}

Additionally, a point should be made about the fact that our participants are not native signers in the sense that they did not acquire ÖGS from birth. The participant group mirrors the language background of the vast majority of ÖGS signers who do not acquire ÖGS from birth, but later in Kindergarten or school with signing peers and/or adults. However, all of the participants use ÖGS as their primary language in daily life and are members of the Deaf community. Age of acquisition is a crucial point, because it has been shown that much later acquisition may influence sign language processing (e.g., Boudreault \& Mayberry, 2006; Malaia \& Wilbur, 2010; Mayberry, Chen, Witcher \& Klein, 2011; Mayberry, Lock \& Kazmi, 2002; Pénicaud et al., 2013) and memory processing of basic word order (e.g., Boudreault \& Mayberry, 2006). It may be useful to note that with respect to the possible effect of age of acquisition on the resolution of locally ambiguous argument structures, in two of our previous studies (Krebs et al., 2018, submitted) a post-hoc analysis of behavioral and ERP data excluding the five latest learners did not show different results in comparison to the analysis including those late learners. Clearly, further investigation is still necessary.

\section{Conclusion}

The present gating study showed that signers preferred SO interpretation when rating locally ambiguous argument structures, which further supports the assumption of a "subject preference" in ÖGS. This observation underlines the claim that the phenomenon of the "subject preference" is a universal (and therefore modality-independent) ambiguity resolution processing strategy, as suggested by Bornkessel-Schlesewsky et al. (2008). Furthermore, in line with previous findings (Krebs, 2013, 2017; Krebs et al., 2018), the present study revealed that in ÖGS disambiguation takes place earlier in time than what would be predicted based on theoretical descriptions of sign onset from sign language grammar. In the majority of the sentences, ambiguity was resolved before the path movement and/or the facing of the disambiguating sign (agreeing verbs or AgrMs) was visible. This finding emphasizes the relevance of non-manual markings and transition phases with respect to the processing of sign language syntax. As with most sign languages, the full system of non-manual markings in ÖGS is not yet fully understood, thus the contributions of each one to the syntax and the semantics remain unknown. Additional investigations of the functions of the observed non-manual markings and manual transitions within sign language grammar and the question of what impact these cues might have on sign language processing are necessary.

\section{Acknowledgements}

We want to thank all Deaf informants taking part in the present study. Special thanks are given to Reinhard Grobbauer for signing the stimulus material. In addition, we really appreciate the useful comments of Brendan Costello who reviewed this manuscript.

\section{Funding}

This research received no specific grant from any funding agency in the public, commercial, or not-for-profit sectors.

\section{Notes}

1. Notation conventions: signs are glossed with capital letters; non-manual markings are indicated by lines above the glosses ("q" indicates non-manual question marking; mm indicates adverbial marking for "with relaxation/enjoyment"); the type of inflection (abbreviated by "I"), that is the aspectual marking is denoted in squared brackets (continuous aspect). 
2. Note that there are also a few simultaneously organized structures in spoken languages (e.g., tones layered on vowels in tone languages).

3. Padden (1983) described a further verb group, the "spatial verbs," that express spatial agreement by movement from source to goal and therefore indicate the relation between locations.

4. Per convention, Deaf with an upper-case $D$ refers to deaf or hard-of-hearing humans who define themselves as members of the sign language community. In contrast, deaf refers to the audiological status of an individual.

5. In a further ERP study using similar transitive ÖGS constructions (i.e., involving agreeing verbs) signed by a different signer, we replicated the finding of the subject preference in ÖGS (see Krebs et al., 2018).

6. This early time point of disambiguation was also revealed by Krebs et al. (2018).

7. Mean durations between these time points are as follows (standard deviations are presented in parentheses):

—Agreeing verbs, SO: $137 \mathrm{~ms}$ (96 ms); OS: $45 \mathrm{~ms}$ (41 ms).

—Plain verbs, SO: $143 \mathrm{~ms}$ (82 ms); OS: $62 \mathrm{~ms}(68 \mathrm{~ms})$.

8. In addition, in most sentences eye-gaze was directed towards the object position, but this non-manual marking mostly occurred at a later time point, namely simultaneously with the path movement of the agreement marking element.

9. Note that, in this study, only regular agreeing verbs moving from subject to object position were included.

10. The final level of a categorical predictor does not receive its own contrast in a regression model, as this would lead to overparameterization.

11. A further difference between the complex signs involving the gender-marking sign (used in the AgrM-BC and the agreeing verbs simultaneous conditions) and the compounds (used in the agreeing verbs serial condition) is that the items with the complex signs appear as first arguments within the sentence. In contrast, in the agreeing verbs with serial referencing, the compounds appear as the second arguments within the sentences.

12. Recall that we assume that, in the one sentence in which the location of the agreeing verb was established in the critical gate, a semantic connotation may have led to a later abandoning of the subject preference.

13. Lexical movement has been divided into two subcomponents: the path movement and the hand-internal movement. Path movement can be straight, arcing, or circular. Internal movement can involve a change in handshape, a change in hand orientation, or a combination of both. Further, path movement and internal movement can occur in combination. Lexical movement has been described as the most salient parameter within signs, and therefore is considered to constitute a syllabic unit of signs, that is, functioning as syllable peaks within signs (Brentari, 1998; Wilbur, 2011).

14. This time-point defined as sign onset, often denoted as "first hold," was first proposed by Newkirk (1977) and Klima and Bellugi (1979). Liddell and Johnson (1989) used this definition of sign onset within their phonological "Hold-Movement-Hold" model, which was adopted/modified by Sandler (1989). Wilbur and Allen (1991) and Wilbur and Petersen (1997) provide two types of experimental evidence against such a three-segment ("Hold-Movement-Hold" style) model.

15. According to Wilbur and Nolen (1986), transitional movements can have syllabic status at the phonetic level, such as for the purposes of counting syllables or tapping, but they are definitely considered "light" syllables (following the definition in Brentari, 1998).

\section{ORCID iD}

Phillip M. Alday (D) https://orcid.org/0000-0002-9984-5745.

\section{References}

Bahan, B. J. (1996). Non-manual realization of agreement in American Sign Language. (Doctoral dissertation). Boston University, Boston.

Barr, D. J., Levy, R., Scheepers, C., \& Tily, H. J. (2013). Random effects structure for confirmatory hypothesis testing: Keep it maximal. Journal of Memory and Language, 68, 255-278. 
Bates, D., Kliegl, R., Vasishth, S., \& Baayen, H. (2015). Parsimonious mixed models. arXiv preprint arXiv:1506.04967.

Bates, D., Mächler, M., Bolker, B., \& Walker, S. (2015). Fitting linear mixed-effects models using lme4. Journal of Statistical Software, 67. https://doi.org/10.18637/jss.v067.i01.

Bornkessel, I., McElree, B., Schlesewsky, M., \& Friederici, A. D. (2004). Multi-dimensional contributions to garden path strength: Dissociating phrase structure from case marking. Journal of Memory and Language, 51, 495-522.

Bornkessel, I., \& Schlesewsky, M. (2006). The extended argument dependency model: A neurocognitive approach to sentence comprehension across languages. Psychological Review, 113, 787-821.

Bornkessel-Schlesewsky, I., Choudhary, K. K., Witzlack-Makarevich, A., \& Bickel, B. (2008). Bridging the gap between processing preferences and typological distributions: Initial evidence from the online comprehension of control constructions in Hindi. In A. Malchukov \& M. Richards (Eds.), Scales (Linguistische ArbeitsBerichte 86) (pp. 397-436). Leipzig, Germany: Institut für Linguistik.

Bornkessel-Schlesewsky, I., \& Schlesewsky, M. (2009). Processing syntax and morphology. A Neurocognitive perspective. Oxford, UK: Oxford University Press.

Boudreault, P., \& Mayberry, R. I. (2006). Grammatical processing in American Sign Language: Age of firstlanguage acquisition effects in relation to syntactic structure. Language and Cognitive Processes, 21, 608-635.

Brentari, D. (1998). A prosodic model of sign language phonology. Cambridge, MA: MIT Press.

Brentari, D. (1989). Backwards verbs in ASL: Agreement re-opened. In L. MacLeod (Ed.), Parasession on agreement in grammatical theory (CLS 24, Vol. 2) (pp. 16-27). Chicago: Chicago Linguistic Society.

Brentari, D., Poizner, H., \& Kegl, J. (1995). Aphasic and Parkinsonian signing: Differences in phonological disruption. Brain and Language, 48, 69-105.

Bross, F., \& Hole, D. (2017). Scope-taking strategies and the order of clausal categories in German Sign Language. Glossa: A journal of general linguistics, 2, 1-30.

Clark, L. E., \& Grosjean, F. (1982). Sign recognition processes in American Sign Language: The effect of context. Language and Speech, 25, 325-340.

Crocker, M. W. (1994). On the nature of the principle-based sentence processor. In C. Clifton, Jr., L. Frazier, \& K. Rayner (Eds.), Perspectives on sentence processing (pp. 245-266). Hillsdale, NJ: Erlbaum.

Emmorey, K., \& Corina, D. P. (1990). Lexical recognition in sign language: Effects of phonetic structure and morphology. Perceptual and Motor Skills, 71, 1227-1252.

Fischer, S. D. (1975). Influences on word order change in American Sign Language. In C. Li (Ed.), Word order and word order change (pp. 1-25). Austin, TX: The University of Texas Press.

Frazier, L., \& Fodor, J. D. (1978). The sausage machine: A new two-stage parsing model. Cognition, 6, 291-325.

Friedman, L. A. (1976). The manifestation of subject, object, and topic in the American Sign Language. In C. N. Li (Ed.), Subject and topic (pp. 125-148). New York: Academic Press.

Gelman, A., \& Stern, H. (2006). The difference between "significant" and "not significant" is not itself statistically significant. The American Statistician, 60, 328-331.

Geraci, C. (2013). Spatial syntax in your hands. Presentation at Language Seminar, CNRS Institut JeanNicod \& DEC, Paris, France.

Gibson, E. (1998). Linguistic complexity: Locality of syntactic dependencies. Cognition, 68, 1-76.

Green, K. (1984). Sign boundaries in American Sign Language. Sign Language Studies, 42, 65-91.

Grosjean, F. (1981). Sign and word recognition: A first comparison. Sign Language Studies, 32, 195-220.

Hanke, T., Matthes, S., Regen, A., \& Worseck, S. (2012, May). Where does a sign start and end? Segmentation of continuous signing. Paper presented at the 5th Workshop on the Representation and Processing of Sign Languages: Interactions between Corpus and Lexicon Language Resources and Evaluation Conference (LREC), Istanbul, Turkey. Retrieved from https://www.researchgate.net/publication/258629159.

Haupt, F. S., Schlesewsky, M., Roehm, D., Friederici, A. D., \& Bornkessel-Schlesewsky, I. (2008). The status of subject-object reanalyses in the language comprehension architecture. Journal of Memory and Language, 59, 54-96.

Hosemann, J., Herrmann, A., Steinbach, M., Bornkessel-Schlesewsky, I., \& Schlesewsky, M. (2013). Lexical prediction via forward models: N400 evidence from German Sign Language. Neuropsychologia, 51, 2224-2237. 
Jaeger, T. F. (2008). Categorical data analysis: Away from ANOVAs (transformation or not) and towards logit mixed models. Journal of Memory and Language, 59, 434-446.

Jantunen, T. (2010). On the role of transitions in signed language. Presentation at the Theoretical Issues in Sign Language Research conference (TISLR), Purdue University, West Lafayette, PA.

Jantunen, T. (2013). Signs and transitions: Do they differ phonetically and does it matter?. Sign Language Studies, 13, 211-237.

Jantunen, T. (2015). How long is the sign? Linguistics, 53, 93-124.

Keenan, E. (1976). Towards a universal definition of "subject." In C. N. Li (Ed.), Subject and topic (pp. 303-333). New York: Academic Press.

Kegl, J. (2004). ASL syntax. Research in progress and proposed research. Sign Language \& Linguistics, 7 , 173-206.

Klima, E. S., \& Bellugi, U. (1979). The signs of language. Cambridge, MA: Harvard University Press.

Krebs, J. (2013). The processing of word order variations in Austrian Sign Language (ÖGS). An ERP-study on subject preference. (Master's Thesis). University of Salzburg, Salzburg, Austria.

Krebs, J. (2017) The syntax and the processing of argument relations in Austrian Sign Language (ÖGS). (Doctoral dissertation). University of Salzburg, Salzburg, Austria.

Krebs, J., Malaia, E., Wilbur, R. B., \& Roehm, D. (2018). Subject preference emerges as cross-modal strategy for linguistic processing. Brain Research, 1691, 105-117.

Krebs, J., Malaia, E., Wilbur, R. B., \& Roehm, D. (submitted). Neural evidence for linguistic status of classifiers in sign language.

Krebs, J., Wilbur, R. B., \& Roehm, D. (2017). Two agreement markers in Austrian Sign Language (ÖGS). Sign Language and Linguistics, 20, 27-53.

Kretzschmar, F., Bornkessel-Schlesewsky, I., Staub, A., Roehm, D., \& Schlesewsky, M. (2012). Prominence facilitates ambiguity resolution: On the interaction between referentiality, thematic roles and word order in syntactic reanalysis. In M. J. A. Lamers, \& P. de Swart (Eds.), Case, word order, and prominence. Interacting cues in language production and comprehension (pp. 239-271). Dordrecht, Netherlands: Springer.

Lepic, R., Börstell, C., Belsitzman, G., \& Sandler, W. (2016). Taking meaning in hand: Iconic motivations in two-handed signs. Sign Language \& Linguistics, 19, 37-81.

Li, C. N., \& Thompson, S. A. (1976). Subject and topic: A new typology of language. In C. N Li (Ed.), Subject and topic (pp. 457-489). New York: Academic Press.

Liddell, S. K. (1980). American Sign Language syntax. The Hague: Mouton de Gruyter.

Liddell, S. K., \& Johnson, R. E. (1989). American Sign Language: the phonological base. Sign Language Studies, 64, 195-277.

Malaia, E., \& Wilbur, R. B. (2010). Early acquisition of sign language: What neuroimaging data tell us. Sign Language \& Linguistics, 13, 183-199.

Malaia, E., Borneman, J. D., \& Wilbur, R. B. (2017). Information transfer capacity of articulators in American Sign Language. Language \& Speech. https://doi.org/10.1177/0023830917708461.

Mayberry, R. I., Chen, J. K., Witcher, P., \& Klein, D. (2011). Age of acquisition effects on the functional organization of language in the adult brain. Brain and Language, 119, 16-29.

Mayberry, R. I., Lock, E., \& Kazmi, H. (2002). Development: Linguistic ability and early language exposure. Nature, 417, 38-38.

Meir, I. 1998. Thematic structure and verb agreement in Israeli Sign Language. (Doctoral dissertation). Hebrew University of Jerusalem, Jerusalem, Israel.

Morford, J. P., \& Carlson, M. L. (2011). Sign perception and recognition in non-native signers of ASL. Language Learning and Development, 7, 149-168.

Neidle, C., Kegl, J., MacLaughlin, D., Bahan, B., \& Lee, R. G. (2000). The syntax of American Sign Language: Functional categories and hierarchical structure. Cambridge, MA: MIT Press.

Newkirk, D. (1977). Surface level handshape assimilation in American Sign Language (A brief description). Unpublished manuscript, The Salk Institute, San Diego.

Padden, C. (1983). Interaction of morphology and syntax in American Sign Language. (Doctoral dissertation). University of California, San Diego, CA [published 1988, New York: Garland Press]. 
Pénicaud, S., Klein, D., Zatorre, R. J., Chen, J. K., Witcher, P., Hyde, K., \& Mayberry, R. I. (2013). Structural brain changes linked to delayed first language acquisition in congenitally deaf individuals. Neuroimage, $66,42-49$.

Pfau, R., \& Steinbach, M. (2006). Modality-independent and modality-specific aspects of grammaticalization in sign languages (Linguistics in Potsdam 24). Potsdam, Germany: Universitäts-Verlag.

Pfau, R., \& Steinbach, M. (2013). Person climbing up a tree (and other adventures in sign language grammaticalization). Sign Language \& Linguistics, 16, 189-220.

Sandler, W. (1989). Phonological representation of the sign: Linearity and nonlinearity in American Sign Language. Dordrecht, Netherlands: Foris.

Sandler, W., \& Lillo-Martin, D. (2006). Sign languages and linguistic universals. Cambridge, UK: Cambridge University Press.

Sapountzaki, G. (2012). Agreement auxiliaries. In R. Pfau, M. Steinbach, \& B. Woll (Eds.), Sign language. An international handbook (pp. 204-227). Berlin, Germany: Mouton de Gruyter.

Schlesewsky, M., Fanselow, G., Kliegl, R., \& Krems, J. (2000). The subject preference in the processing of locally ambiguous wh-questions in German. In B. Hemforth, \& L. Konieczny (Eds.), German sentence processing (pp. 65-93). Dordrecht, Netherlands: Kluwer.

Skant, A., Dotter,F., Bergmeister,E.,Hilzensauer, M.,Hobel, M., Krammer, ... Unterberger, N.(2002). Grammatik der Österreichischen Gebärdensprache [The grammar of Austrian Sign Language]. Klagenfurt, Austria: Veröffentlichungen des Forschungszentrums für Gebärdensprache und Hörgeschädigtenkommunikation der Universität Klagenfurt [Publications of the Research Center for wSign Language and Hearing Impaired Communication of the University of Klagenfurt] (Vol. 4).

Steinbach, M., \& Pfau, R. (2007). Grammaticalization of auxiliaries in sign languages. In P. Perniss, R. Pfau, \& M. Steinbach (Eds.), Visible variation: Comparative studies on sign language structure (pp. 303-339). Berlin, Germany: Mouton de Gruyter.

Ten Holt, G. A., van Doorn, A. J., de Ridder, H., Reinders, M. J. T., \& Hendriks, E. A. (2009). Which fragments of a sign enable its recognition? Sign Language Studies, 9, 211-239.

Thompson, R., Emmorey, K., \& Kluender, R. (2006). The relationship between eye gaze and verb agreement in American Sign Language: An eye-tracking study. Natural Language \& Linguistic Theory, 24, 571-604.

Wilbur, R. B. (1990). An experimental investigation of stressed sign production. International Journal of Sign Linguistics, 1, 41-59.

Wilbur, R. B. (2000). Phonological and prosodic layering of nonmanuals in American Sign Language. In H. Lane, \& K. Emmorey (Eds.), The signs of language revisited: Festschrift for Ursula Bellugi and Edward Klima (pp. 213-241). Hillsdale, NJ: Erlbaum.

Wilbur, R. B. (2002). Phrase structure in ASL and ÖGS. In R. Schulmeister, \& H. Reinitzer (Eds.), Progress in sign language research. In honor of Siegmund Prillwitz (pp. 235-247). Hamburg, Germany: Signum.

Wilbur, R. B. (2005). Evidence from ASL and ÖGS for asymmetries in UG. In A. M. DiScuillo (Ed.), UG and external systems: Language, brain and computation (pp. 193-210). Amsterdam, Netherlands: John Benjamins.

Wilbur, R. B. (2009). Productive reduplication in a fundamentally monosyllabic language. Language Sciences, 31, 325-342.

Wilbur, R. B. (2011). Sign syllables. In M. van Oostendorp, C. J. Ewen, E. V. Hume, \& K. Rice (Eds.), The Blackwell companion to phonology (Vol. 1) (pp. 1309-1334). Chichester, UK: Wiley-Blackwell.

Wilbur, R. B. (2015). 126. Word-formation and sign languages. In P. O. Müller, I. Ohnheiser, S. Olsen, \& F. Rainer (Eds.), Word-formation: An international handbook of the languages of Europe (Vol. 3) (pp. 2225-2251). Berlin, Germany: De Gruyter Mouton.

Wilbur, R. B. (2018). Production of signed utterances. In E. M. Fernández, \& H. M. I. Cairns (Eds.), Handbook of psycholinguistics (pp. 111-135). Hoboken, NJ: Wiley-Blackwell.

Wilbur, R. B., \& Allen, G. D. (1991) Perceptual evidence against internal structure in ASL syllables. Language \& Speech, 34, 27-46.

Wilbur, R. B., \& Nolen, S. B. (1986). The duration of syllables in American sign language. Language \& Speech, 29, 263-280.

Wilbur, R. B., \& Petersen, L. (1997). Backwards signing and ASL syllable structure. Language \& Speech, 40, 63-90. 


\section{Appendix}

Plots of mean ratings for subcategories:

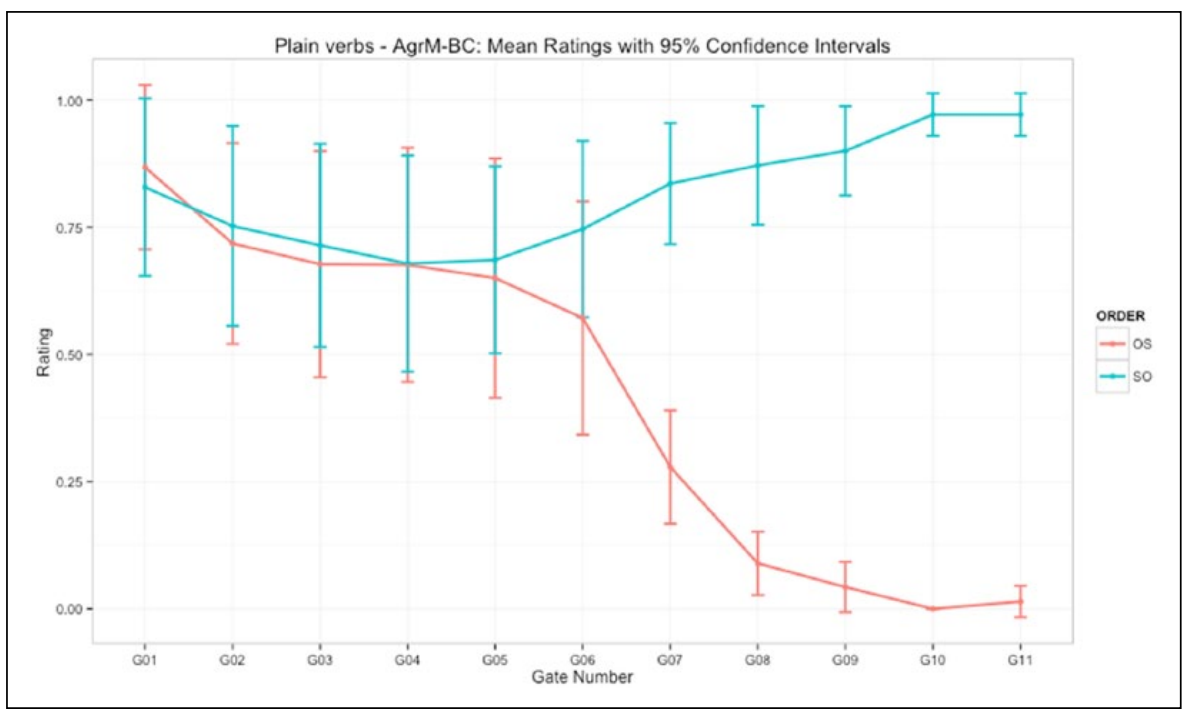

Comparison of SOV and OSV orders for the subcategory plain verbs - AgrM-BC. Gate numbers are given on the $\mathrm{x}$-axis; mean ratings, i.e. the proportion of sentences interpreted as $\mathrm{SO}$ are presented on the $\mathrm{y}$-axis. The relevant gate appeared at gate 7 .

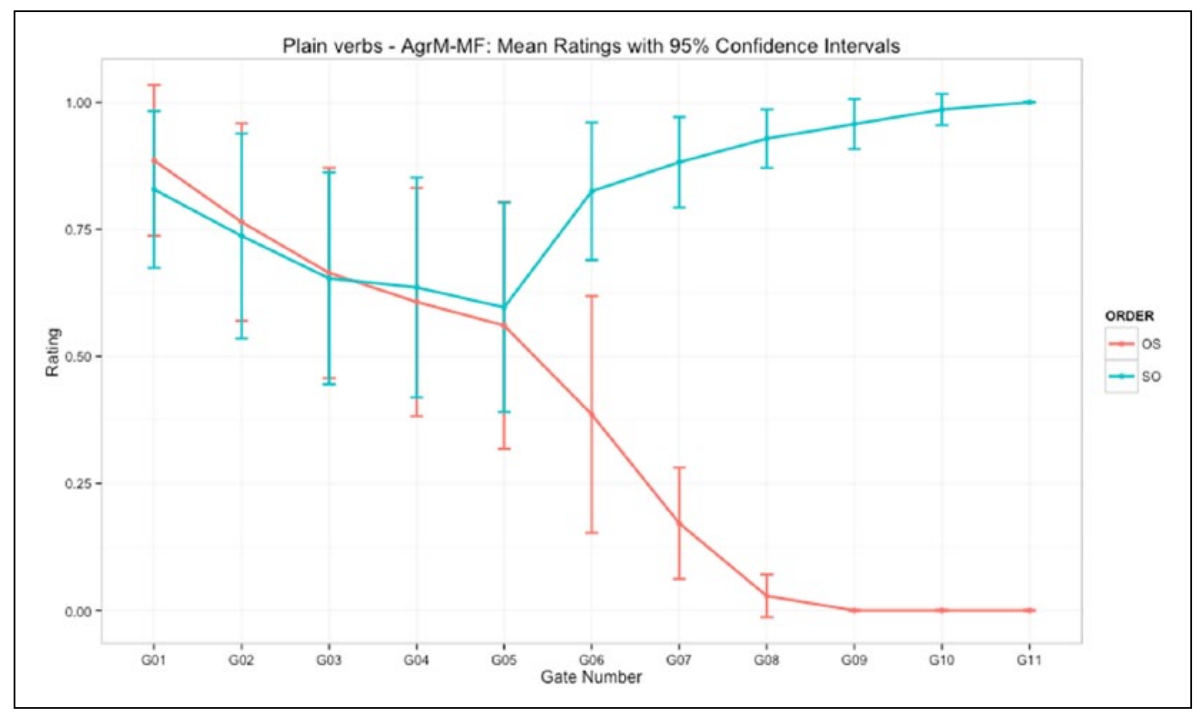

Comparison of SOV and OSV orders for the subcategory plain verbs - AgrM-MF. Gate numbers are given on the $\mathrm{x}$-axis; mean ratings, i.e. the proportion of sentences interpreted as $\mathrm{SO}$ are presented on the $\mathrm{y}$-axis. The relevant gate appeared at gate 6 . 


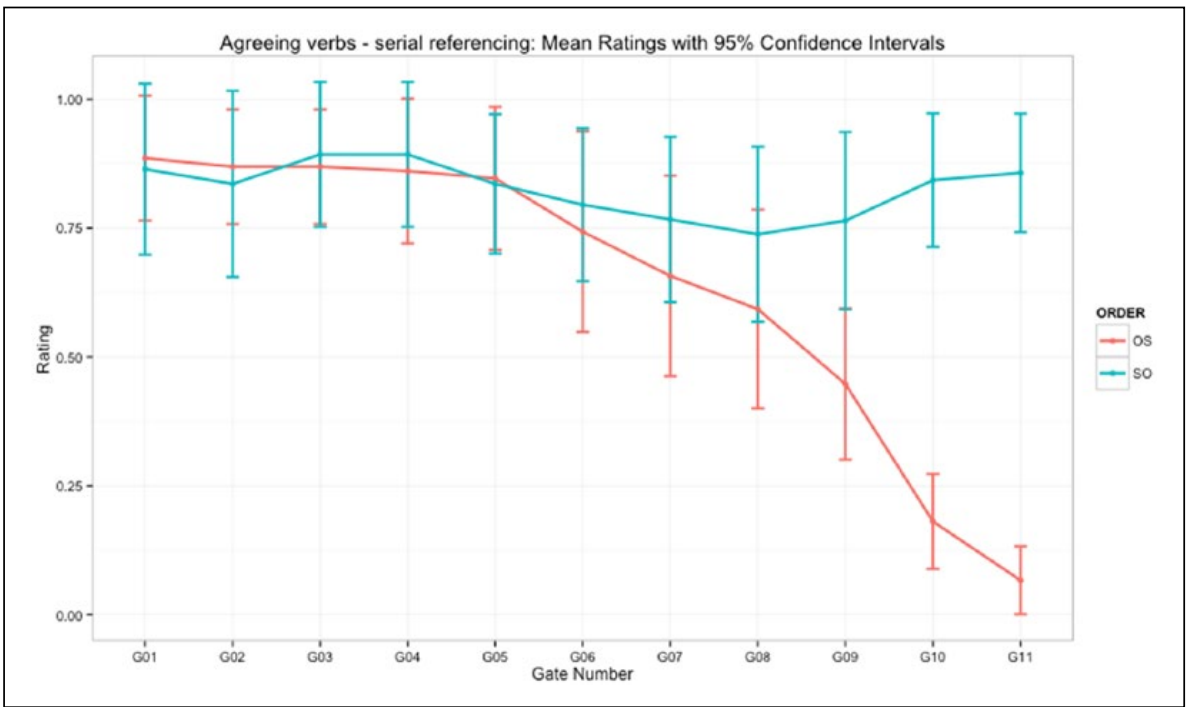

Comparison of SOV and OSV orders for the subcategory agreeing verbs - serial referencing. Gate numbers are given on the $\mathrm{x}$-axis; mean ratings, i.e. the proportion of sentences interpreted as $\mathrm{SO}$ are presented on the $y$-axis. The relevant gate appeared at gate 9 .

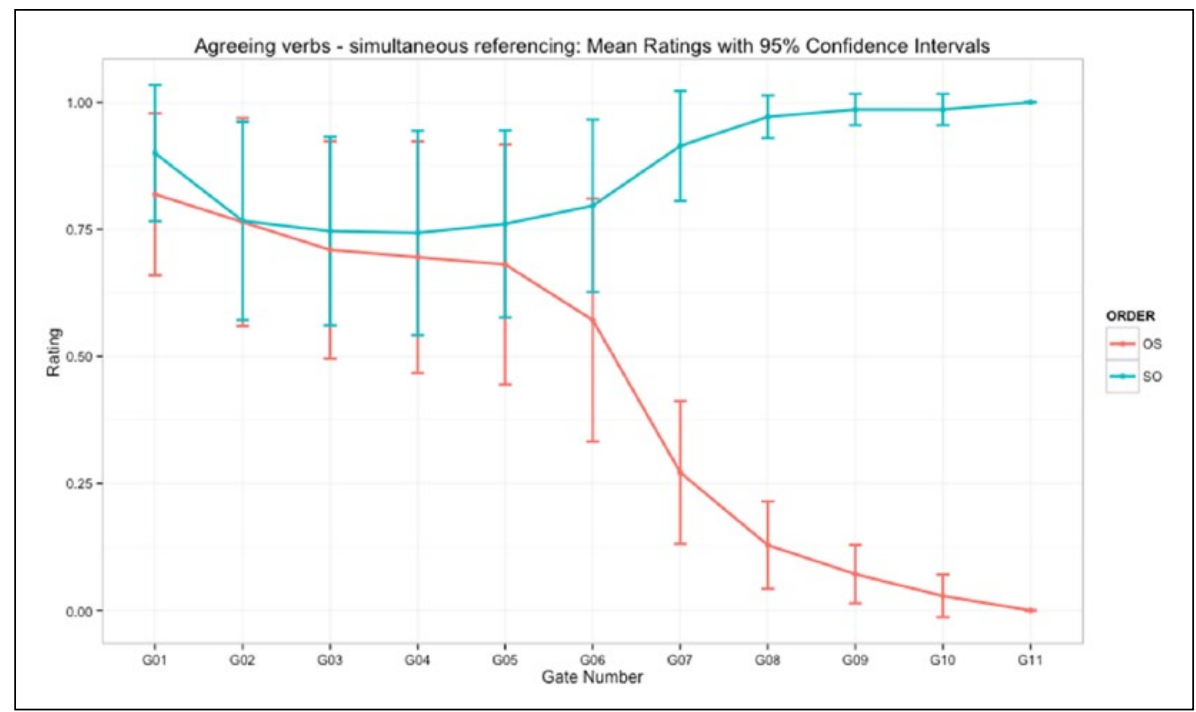

Comparison of SOV and OSV orders for the subcategory agreeing verbs - simultaneous referencing. Gate numbers are given on the $\mathrm{x}$-axis; mean ratings, i.e. the proportion of sentences interpreted as $\mathrm{SO}$ are presented on the $y$-axis. The relevant gate appeared at gate 7. 